\title{
Applications of multi-variate analysis of variance (MANOVA) to multi-electrode array electrophysiology data
}

\author{
P.M. Horton ${ }^{\mathrm{a}, *}$, L. Bonny ${ }^{\mathrm{a}}$, A.U. Nicol ${ }^{\mathrm{b}}$, K.M. Kendrick ${ }^{\mathrm{b}}$, J.F. Feng ${ }^{\mathrm{c}, \mathrm{d}, * *}$ \\ ${ }^{a}$ Department of Informatics, Sussex University, Falmer, Brighton BN1 9QH, UK \\ ${ }^{\mathrm{b}}$ Laboratory of Cognitive and Behavioural Neuroscience, The Babraham Institute, Cambridge CB2 4AT, UK \\ ${ }^{\mathrm{c}}$ Department of Mathematics, Hunan Normal University, 410081, Changsha, PR China \\ ${ }^{\mathrm{d}}$ Department of Computer Science, Warwick University, Coventry CV4 7AL, UK
}

Received 11 August 2004; received in revised form 22 November 2004; accepted 13 January 2005

\begin{abstract}
We have developed an adaptation of multi-variate analysis of variance (MANOVA) to analyze statistically both local and global patterns of multi-electrode array (MEA) electrophysiology data where the activities of many (typically >100) neurons have been recorded simultaneously. Whereas simple application of standard MANOVA techniques prohibits extraction of useful information in this kind of data, our new approach, MEANOVA (=MEA + MANOVA), allows a more useful and powerful approach to analyze such complex neurophysiological data. The MEANOVA test enables the detection of the "hot-spots" in the MEA data and has been validated using recordings from the rat olfactory bulb. To further validate the power of this approach, we have also applied the MEANOVA test to data obtained from a simple computational network model. This MEANOVA software and other useful statistical methods for MEA data can be downloaded from http://www.sussex.ac.uk/Users/pmh20.
\end{abstract}

(C) 2004 Elsevier B.V. All rights reserved.

Keywords: Multi-electrode array; Multi-variate statistical analysis; Olfactory bulb; Odour; Olfactory bulb modelling

\section{Introduction}

After over a century of neurophysiological research, we still do not understand the principle by which a stimulus, such as an odour, an image or a sound, is represented by distributed neural ensembles within the brain (Albright et al., 2000; Dayan and Abbott, 2001; Feng, 2004). While large numbers of studies have made detailed analyses of response profiles of single cells in isolation, such techniques cannot easily address holistic issues of how large ensembles of neurones can integrate information both spatially and temporally. There is little doubt that much of the information processing power of the brain resides in the activities of co-operating and competing networks of neurones and that if we can unlock the principles whereby information is encoded within these networks

\footnotetext{
* Corresponding author. Tel.: +44 7963 246444; fax: +44 1273877873.

** Co-corresponding author.

E-mail addresses: pmh20@ sussex.ac.uk (P.M. Horton), jianfeng@sussex.ac.uk (J.F. Feng)
}

as a whole, rather than within single neurones in isolation, we may actually be able to understand how the brain works.

While some progress towards understanding how this is achieved at a gross structural level is being achieved with brain imaging techniques, a promising approach to provide an understanding at the level of multiple cell-cell interactions in the brain is the simultaneous recording of the output (action potentials, spikes) from large numbers of cells individually within a defined system. This technology has been applied to an expanding number of studies of neural activity, in both perceptual (Maynard et al., 1999) and cognitive (Johnson and Welsh, 2003) systems, and also in the development of implantable neuroprosthetic devices (Patterson et al., 2004; Warwick et al., 2004). The two main difficulties in achieving this step have been, firstly, the lack of appropriate tools to make simultaneous recordings from large numbers of individual neurones within a network and, secondly, the issue of analysing the resulting huge amount of high-density multi-variate data, in which significant effects may be sparse across the large sample of neurons. 
A small number of groups have now developed the necessary recording hardware and software tools for data acquisition and these are available commercially (e.g. Plexon Inc. and Cyberkinetics Inc., USA). Currently, however, even with some data compression algorithms, recordings from 128 electrodes sampled at $100 \mathrm{kHz}$ routinely produce around $15 \mathrm{MB}$ of data/min. With most individual experimental sessions lasting several hours or more this can produce data sets of many hundreds of GB. There are a number of software analytical packages for editing and visualising data using raster/event plots or pseudocolour grids, and it is possible to provide a relatively cursory analysis of this high volume data using generic programmes (e.g. Matlab, NeuroExplorer, Spike2). However, there is very little that has been developed for detailed statistical analysis, and subsequent visual representation of the changing spatial and temporal patterns of information recorded across the electrode arrays.

The multi-electrode array (MEA), used in our experiments for recording of the olfactory bulb (OB), records an area of neural activity by using several electrodes. Each electrode records activity of a subset of the whole area. From this, we can examine the whole area of neural activity to see the overall affect of applying an external factor, i.e. a stimulus, or to look at subsets of the area, to find if many or individual clusters of neurons are responding to the external factor.

A graphic representation of a typical MEA used in experiments is shown in Fig. 1. Usually, in a single experiment, of the total number of electrodes $(\mathcal{Q})$, only a proportion (a subset of $\mathcal{Q}$ ) will actually sample discriminable spikes from single neurones. For the simplicity of notation, we do not distinguish between the subset and $\mathcal{Q}$.

The most straightforward method to compare or assess the difference between two sets of multi-variate random variables is multi-variate analysis of variance (MANOVA) (Johnson and Wichern, 1988). Under the normal distribution assumption, we can, in theory, directly apply MANOVA to MEA data. Nevertheless, within an MEA recording, we would naturally expect that only a variable proportion of cell activities

\section{MEA}

- Dorsal

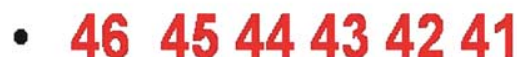

- 363534333231

- 262524232221

- 161514131211

- 060504030201 caudal

- Ventral

Fig. 1. A typical 30 electrode array $(5 \times 6$ electrodes $)$ used in our experiments to record activity in the rat olfactory bulb. $\mathcal{Q}=\{01,02,03$, $\ldots, 45,46\}$. recorded by the electrodes in the array would respond to a stimulus. In other words, the activity changes of some cells are informative whereas those of others (which could be the majority) are not. If we simply include all activities from both responsive and non-responsive cells in a MANOVA test, the significance of data from the former might be completely obscured or diluted by the latter. The net result of this could be that even though a small number of the cells recorded in a neuronal ensemble could each be shown by simple paired $t$-tests to exhibit significant changes in firing rate in response to stimulus presentation, the proper application of MANOVA (taking into account all the population variables recorded) would assert that there were no stimulus-evoked changes.

In the current approach, we have overcome this difficulty by estimating a significance score to individual, and subsets of electrodes (which are geometrically connected). The layout of the electrodes can be seen in Fig. 1. The outcome of the analysis will conclude whether the area of neurons covered by the multi-electrode array has responded significantly to a factor, i.e. a stimulus. The initial stage in the analysis is to analyse individual, and then groups of electrodes, until the last group examined contains all of the electrodes in the array that have sample neuronal activity. For example, if electrodes 01,02 and 03 only recorded activity, they would firstly be analysed individually. The next stage is to analyse the integration of 01 and 02 , then 02 and 03 . The final analysis then, would be to examine the recordings of all three electrodes together. These six tests will result in six scores, which will refer to where in the recorded area, changes in neuronal activity are, thus finding the "hot-spots" of the data. The bigger the score associated with an area, the more significant the change in neural activity, i.e. if one electrode area showed a bigger score than the others, this would conclude that this area of the MEA has had the biggest change in activity, thus being the most significant.

The applicability of this novel approach is tested here using both MEA data obtained from computational modelling and data recorded from the rat olfactory bulb during odour stimulation.

\section{Analytical methods}

\subsection{Normal distribution}

The first requirement for carrying out a MEANOVA test is to check whether the data is normally distributed or not. If spikes of each neuron are independent then, according to the renewal theorem (Feller, 1971), we know that

$\frac{N_{t}}{t} \rightarrow N\left(\mu, \frac{\sigma^{2}}{\mu^{3}}\right) \quad$ (normal distribution) as $t \rightarrow \infty$

\footnotetext{
${ }^{1}$ The term "hot-spot" is defined in this paper as an area or areas of neurons which has had a significant change in activity, in response to a factor, e.g. a stimulus.
} 
where $\mu$ is the mean interspike interval, $\sigma^{2}$ the variance and $N_{t}$ is the spike counting inside time window $[0, t]$. The independent assumption can be relaxed and replaced by a weak assumption: the spike train is stationary. Of course, the stationary assumption is still a strong requirement for many cases. For example, in the MEA data from the olfactory bulb, it is clear that habituation is a common phenomenon and occurs over an even smaller time scale than the recording period used for analysis (5-10 s). There are a few ways to overcome the difficulty. For example, when we calculate $N_{t}$, we could introduce weights for different interspike intervals, a typical de-trend method, and so on.

Of course, in the data from our modelling approach, since the interspike intervals are generated from an integrate-andfire model, the interspike intervals are perfectly renewal processes. Hence, all our results hold true. For multi-dimensional renewal theorem, we refer the reader to Hunter (1974a,b).

For experimental data, as far as we can tell, all statistical approaches including the one we developed here are valid only to a certain degree. For example, even for an in vivo recording from a single cell, we know its output is no longer a stationary process. Hence, the assumption for a routine statistical test such as $t$-test or Komogrov test fails.

However, up to date it is difficult, if not impossible, to simulate the local field potential, since we do not know the exact sources to generate it; hence, we can not check the final results using modelling approach. Here, we only use it on spiking data.

\subsection{Two-way MANOVA}

For a particular experiment, for example, with the first recording trial with exposure to a fixed concentration of odour, let us assume that we have $\mathcal{Q}$ electrodes in the array and have recorded the activity of $P$ cells. Let $Y=$ $\left(y_{p, q}, p=1, \ldots, P\right), q \in \mathcal{Q}$ are spike trains with $y_{p, q}=$ $\left(t_{p, q}^{k}, k=1, \ldots, K_{p, q}\right)$, where $t_{p, q}^{k}$ is the $k$ th spike and $K_{p, q}$ is the total number of spikes within the recorded time window $T$. For a given bin size $t$, we can divide $T$ into $M=T / t$ time bins. Inside each time bin, let $N_{p, q}^{m}$ represent the spike counting of the $m$ th window, $m=1, \ldots, M=T / t$. Hence, we have

$\mathcal{N}^{m}=\left\{N_{p, q}^{m}, m=1, \ldots, T / t, p=1, \ldots, P\right\}, \quad q \in \mathcal{Q}$

which is a normally distributed, $p$-dimensional vector. In other words, we have a random vector $\mathcal{N}$ with $P$ dimensions and $M=T / t$ samplings (replicates).

The random vector $\mathcal{N}^{m}$ is usually dependent on various other factors. If we have two sampling periods (pre- and during-stimulus presentation) over three trials, we have $i=1$ (pre-stimulus) and $i=2$ (during stimulus), $j=1$ (trial 1), $j=2$ (trial 2) and $j=3$ (trial 3). For each fixed $i, j$ and $m$, we have

$$
X_{i j m}=\mu+F_{i}^{1}+F_{j}^{2}+F_{i j}^{1,2}+\xi_{i j m}
$$

where $\mu$ is the mean vector, $F^{1}$ the effect of the first factor, for example, stimulus factor, $F^{2}$ the effect of the second factor, for example, trial factor, $F^{1,2}$ the interaction between the two factors, $\xi$ a normally distributed random vector with mean zero, $i=1, \ldots, I, j=1, \ldots, J$ and $m=1, \ldots, M$ is the number of samplings. In other words, $X_{i j m}$ is a sampling of $\mathcal{N}^{m}$, that is, $X_{i j m}$ is the array activity of $m$ th window. Note that $X_{i j m}$ is a $P$-dimensional random vector. For simplicity of notation, let us further introduce that

$$
\begin{aligned}
& \bar{X}_{i \cdot}=\sum_{j=1}^{J} \sum_{m=1}^{M} \frac{X_{i j m}}{J M}, \\
& \bar{X}_{\cdot j}=\sum_{i=1}^{I} \sum_{m=1}^{M} \frac{X_{i j m}}{I M}, \\
& \bar{X}_{i j}=\sum_{m=1}^{M} \frac{X_{i j m}}{M}, \\
& \bar{X}=\sum_{i=1}^{I} \sum_{j=1}^{J} \sum_{m=1}^{M} \frac{X_{i j m}}{I J M}
\end{aligned}
$$

where $\bar{X}$ is the average of all the observations, $\bar{X}_{i}$. the average of the observation vectors at the $i$ th level of factor $1, \bar{X}_{. j}$ the average of the observation vectors at the $j$ th level of factor 2 and $\bar{X}_{i j}$ is the average of the observation vectors at the $i$ th level of factor 1 and the $j$ th level of factor 2. From Eqs. (2.2) to (2.5), we can decompose the equation above in (2.1) to describe $X_{i j m}$ the observational vector, using the newly defined variables.

$$
\begin{aligned}
X_{i j m}= & \bar{X}+\left(\bar{X}_{i \cdot}-\bar{X}\right)+\left(\bar{X}_{\cdot j}-\bar{X}\right) \\
& +\left(\bar{X}_{i j}-\bar{X}_{i \cdot}-\bar{X}_{\cdot j}+\bar{X}\right)+\left(X_{i j m}-\bar{X}_{i j}\right)
\end{aligned}
$$

This equation shows how the observed result is achieved by examining the factors which have contributed to get the found result, i.e. how much factor $1\left(\bar{X}_{i .}-\bar{X}\right)$, factor $2\left(\bar{X}_{\cdot j}-\bar{X}\right)$, interaction $\left(\bar{X}_{i j}-\bar{X}_{i \cdot}-\bar{X}_{\cdot j}+\bar{X}\right)$ and the remainder of the variance (residual error) $\left(X_{i j m}-\bar{X}_{i j}\right)$ have contributed to the total variance, thus resulting in the observation. The interaction describes the relationship between the various levels of factors 1 and 2. An example of interaction would be, if a stimulus was presented in three trials, trials 1 and 3 could show an increase in activity but a decrease was shown in trial 2. If this was the case, it would be very difficult to conclude what the affect of the factors were.

The residual error describes the variance which is not accountable by either factor 1 or 2 . If a stimulus was presented and the result of this was that the variance of the residual error was large and the variances of factors 1 and 2 were small, would conclude that the chances are that the observation recorded has been caused by unknown (random) factors and not by either of the factors under examination. 


\subsection{MEANOVA $=$ MEA + MANOVA}

According to the classical MANOVA (Johnson and Wichern, 1988), we see that we should calculate the following matrices:

$$
\begin{aligned}
S_{1}= & \sum_{i=1}^{I} J M\left(\bar{X}_{i \cdot}-\bar{X}\right)\left(\bar{X}_{i \cdot}-\bar{X}\right)^{\prime} \\
S_{2}= & \sum_{j=1}^{J} I M\left(\bar{X}_{\cdot j}-\bar{X}\right)\left(\bar{X}_{\cdot j}-\bar{X}\right)^{\prime} \\
S_{3}= & \sum_{i=1}^{I} \sum_{j=1}^{J} M\left(\bar{X}_{i j}-\bar{X}_{i \cdot}-\bar{X}_{\cdot j}+\bar{X}\right) \\
& \times\left(\bar{X}_{i j}-\bar{X}_{i \cdot}-\bar{X}_{\cdot j}+\bar{X}\right)^{\prime} \\
S_{r}= & \sum_{i=1}^{I} \sum_{j=1}^{J} \sum_{m=1}^{M}\left(X_{i j m}-\bar{X}_{i j}\right)\left(X_{i j m}-\bar{X}_{i j}\right)^{\prime}
\end{aligned}
$$

where $S_{1}$ is the residual error due to the first factor, $S_{2}$ the residual error due to the second factor, $S_{3}$ the residual error due to the interaction between the first factor and the second factor and $S_{r}$ is the total residual. The next stage in the interpretation is to test the likelihood that any of the factors (interactions, factor 1 or 2) caused a change in neuron activity. This is acheived by using Wilks' lambda.

$$
\begin{aligned}
& \Lambda_{3}=\frac{\left|S_{r}\right|}{\left|S_{3}+S_{r}\right|} \quad \Lambda_{1}=\frac{\left|S_{r}\right|}{\left|S_{1}+S_{r}\right|} \\
& \Lambda_{2}=\frac{\left|S_{r}\right|}{\left|S_{2}+S_{r}\right|} .
\end{aligned}
$$

Intuitively, when $\Lambda_{i}, i=1,2,3$, is small, the factor (factor 1,2 or the interaction) is important and so we can reject the hypothesis that the factor is negligible. This is exactly the case in MANOVA.

For large samples, Wilks' lambda can be used with a chisquared percentile, to provide us with confidence that the change in the results was due to the factors, i.e. a stimulus and not by chance. Using Barletts multiplier (Bartletts, 1954), to improve the chi-squared approximation, we reject the hypothesis that there are no significant changes in interactions if

$$
\begin{aligned}
& -\left|I J(M-1)-\frac{P+1-(I-1)(J-1)}{2}\right| \\
& \quad \times \ln \Lambda_{3}>\chi_{(I-1)(J-1) P}^{2}(\alpha)
\end{aligned}
$$

where $\chi_{(I-1)(J-1) P}^{2}(\alpha)$ is the upper $(100 \alpha)$ percentile of a chisquared distribution with $(I-1)(B-1) P(\alpha)$ d.f. The significance level $(\alpha)$ of $5 \%$ was used for all interaction tests.

Under the circumstances that Eq. (2.12) is not true, i.e. the interaction is not strong enough. We can then test for the factors 1 and $2 .^{2}$ We reject the hypothesis that there is no significant change due to factor 1 if

$$
-\left|I J(M-1)-\frac{P+1-(I-1)}{2}\right| \ln \Lambda_{1}>\chi_{(I-1) P}^{2}(\alpha),
$$

where $\chi_{(I-1)(J-1) P}^{2}(\alpha)$ is the upper $(100 \alpha)$ percentile of a chisquared distribution with $(I-1) P(\alpha)$ d.f. The significance level $(\alpha)$ of $5 \%$ was used for all factor 1 tests.

We reject the hypothesis that there are no significant changes due to factor 2 if

$-\left|I J(M-1)-\frac{P+1-(J-1)}{2}\right| \ln \Lambda_{2}>\chi_{(J-1) P}^{2}(\alpha)$.

where $\chi_{(J-1) P}^{2}(\alpha)$ is the upper $(100 \alpha)$ percentile of a chisquare distribution with $(I-1) p(\alpha)$ d.f. The significance level $(\alpha)$ of $5 \%$ was used for all factor 2 tests.

Unfortunately, the text book version of MANOVA can not be directly applied to our MEA data. For example, as shown in Fig. 10 bottom panel right, if we simply put all recorded neurons in our analysis, we will simply conclude that there is no significant change between pre- and during stimulus (see below for more details). The other reason is that we intend to detect the hottest spot (group of electrodes) in experiments, i.e. the group of neurons most significantly changed in response to stimulus or due to other factors.

With the above considerations in mind, we can define a significant score for a subset $\mathcal{S}$ of $\mathcal{Q}$ as

$$
\begin{aligned}
S_{3}(\mathcal{S})= & -\left|I J(M-1)-\frac{|\mathcal{S}|+1-(I-1)(J-1)}{2}\right| \\
& \times \frac{\ln \Lambda_{3}}{\chi_{(I-1)(J-1)|\mathcal{S}|}^{2}(\alpha)}, \\
S_{1}(\mathcal{S})= & -\left|I J(M-1)-\frac{|\mathcal{S}|+1-(I-1)}{2}\right| \\
& \times \frac{\ln \Lambda_{1}}{\chi_{(I-1)|\mathcal{S}|}^{2}(\alpha)}, \\
S_{2}(\mathcal{S})= & -\left|I J(M-1)-\frac{|\mathcal{S}|+1-(J-1)}{2}\right| \\
& \times \frac{\ln \Lambda_{2}}{\chi_{(J-1)|\mathcal{S}|}^{2}(\alpha)},
\end{aligned}
$$

where $|\mathcal{S}|$ is the number of neurons recorded by electrodes in $\mathcal{S}$. It is routinely required that $I J(M-1)>|\mathcal{S}|$.

Please note that the significant score is made up from dividing the improved Wilks' lambda with the test statistic

\footnotetext{
2 One-way MEANOVA is available at http://www.sussex.ac.uk/ Users/pmh20.
} 
drawn from the $\chi^{2}$ distribution. A score of 1 and over will be deemed as significant at the $5 \%$ significance level, i.e. we have 95\% confidence that the results, which have been affected are due to one of the observed factors such as a presented stimulus. The higher the significance score the bigger the change in results, i.e. the bigger the change in neural activity.

Therefore, a subset $\mathcal{S}$ which attains the highest value of the significant score is the 'hottest spot' in the array. The hottest spots reveal the groups of neurons with most significant changes.

\section{Modelling approach}

An olfactory bulb model was created, adopted from a simple model $^{3}$ (Margrie and Schaefer, 2003), for the main purpose of proving that the MEANOVA approach works. To prove this, particular mitral cells (MCs) are chosen to increase their activity in response to stimulus presentation, so the cells responding most significantly will already be known to us. MEANOVA will then be used on the spiking data created from the cells, to see if it yields the expected results, i.e. the chosen and surrounding mitral cells should have the most significant change in activity.

To achieve this, two sets of mitral cell data are needed, to show the activity of the cells, before and during-stimulus presentation. These two sets of data can then be used by MEANOVA to calculate the significant scores.

Three trials will be used, with the activity of the cells being similar in each, and the mitral cells chosen as responsive remaining unchanged throughout. This is implemented to show that in different trials the same neurons change their activity, in response to stimulus presentation. MEANOVA would be used to help prove or disprove this, i.e. if the interaction scores are below 1, it has been shown that particular neurons respond to a particular stimulus. The following two subsections, discuss the model used, and the results gained.

\subsection{Network model used}

Simulations were created and performed in Matlab 6.5 (The MathWorks, Natick, MA, USA). The network consists of a connected set of mitral cells where each mitral cell is connected to all of the adjacent ones. Mitral cells were modelled as leaky integrate-and-fire neurons, the equation is shown below:

$\tau_{\mathrm{m}} \cdot \frac{\mathrm{d} V}{\mathrm{~d} t}=E_{1}-V-g_{\mathrm{s}} \cdot P_{\mathrm{s}} \cdot\left(V-E_{\mathrm{s}}\right)+R_{\mathrm{m}} \cdot I_{\mathrm{e}}+$ noise

The cells had an action potential threshold of $15 \mathrm{mV}$, resting potential $\left(E_{1}\right)$ of $12 \mathrm{mV}, \tau_{\mathrm{m}}=10 \mathrm{~ms}$ and noise was in the range of $[0,0.005] \mathrm{nA} . R_{\mathrm{m}}$ had the value of $1 \mathrm{M} \Omega$.

\footnotetext{
${ }^{3}$ We have developed biophysical models in Davison et al. (2003).
}

The input $\left(I_{\mathrm{e}}\right)$ is in the form of:

$I_{\mathrm{e}}=4 \mathrm{~Hz}$ oscillation current + fixed current

A $4 \mathrm{~Hz}$ oscillatory current is injected into each of the mitral cells with a peak amplitude of $125 \mathrm{pA}$. This mimicked several sniff cycles. Each mitral cell also had a constant current, chosen randomly in the range of $[0.265,0.27] \mathrm{nA}$ at the outset of the experiment injected into it. Thus, each mitral cell differed slightly from the rest. The range needed to be small, to make all trials similar. This would ensure, that the hot areas, across the array would be similar across trials. With a larger range, the random inputs to each neuron in each trial, could vary greatly, thereby influencing the final interaction results, i.e. producing results larger than 1 .

The final input to all mitral cells, is a postsynaptic current. The strength of the connection (conductance) corresponds to the value of $g_{s}$. All connection strengths for all mitral cells were $5 \mathrm{~S}$ (siemens). To produce an inhibitory effect on the connection, reversal potential $\left(E_{\mathrm{s}}\right)$ was set to $-10 \mathrm{mV}$. For an excitatory connection, this was set to $35 \mathrm{mV}$.

Each mitral cell was connected to eight surrounding cells, for example in Fig. 1, cell 23 is connected to cells 12, 13, $14,22,24,32,33$ and 34 . Non-target cells which are not connected to the target cells have reciprocal inhibitory connections. Non-target cells which are connected to a target cell have either an excitatory connection to them from the target cell, forming a cluster of heightened activity around the target, or an inhibitory connection to produce the opposite effect. Target cells always receive an excitatory connection.

The synaptic open probability $\left(P_{\mathrm{S}}\right)$ variable was modelled using the following:

$P \rightarrow P_{\mathrm{s}}+P_{\max }\left(1-P_{\mathrm{s}}\right)$

This is the state of $P_{\mathrm{S}}$ after a pre-synaptic action potential has occurred, $P_{\max }$ having the value of 1 . Immediately after this, the variable decays exponentially to zero, using the following equation:

$\tau_{\mathrm{s}} \cdot \frac{\mathrm{d} P_{\mathrm{s}}}{\mathrm{d} t}=-P_{\mathrm{s}}$

where $\tau_{\mathrm{s}}$ is the time constant for the connection. The excitatory and inhibitory values for the connections was 2 and $6 \mathrm{~ms}$, respectively.

For the purposes of MEANOVA, in this section, one electode corresponds to a single mitral cell. Each electrode has the same labelling and layout as shown in Table 1. The spiking activity of all the cells was recorded over a range of time intervals, and a varying number of time bins. To create a stimulus, another input was injected into the chosen mitral cells.

The stimulus starts from time $t$ at $0 \mathrm{nA}$ and steadily increases over a time period of $(t+$ duration $) / 2$ to a peak of $25 \mathrm{nA}$. The stimulus then steadily decreases to $0 \mathrm{nA}$ where 
Table 1

This table shows the neurons in the a $4 \times 4$ layout

\begin{tabular}{llll}
\hline 01 & 02 & 03 & 04 \\
11 & 12 & 13 & 14 \\
21 & 22 & 23 & 24 \\
31 & 32 & 33 & 34 \\
\hline
\end{tabular}

The target neurons are shown in italic.

the final time of $t+$ duration is reached (duration for this paper was $0.5 \mathrm{~s}$ ). This was used to simulate an increase and decrease of odour concentration. This was used to induce hot-spots, i.e. a change in activity, into the selected mitral cells.

Two experiments were constructed to show the MEANOVA results, for a small and a large group of data, i.e. a small and a large array of neurons.

1. The number of mitral cells used to gain the results in the first experiment was 16, i.e. a $4 \times 4$ array. This is shown in Table 1.

2. The second experiment used a $9 \times 9$ array, and shows that MEANOVA can pick out a few significantly changed neurons from a very large group. The stimulus is also decreased to a peak of $20 \mathrm{nA}$, to show the affects it has on the significant scores.

\subsection{Results generated from model for first experiment}

\subsubsection{Activity of mitral cells}

The activity of the mitral cells when there is no stimulus is shown in Fig. 2 (left). Each square in the grid represents the average activity of one mitral cell. The activity of all the cells is in the range of $5-12 \mathrm{~Hz}$. The firing rates of the cells were similar in all trials (mitral cell activity in trials 2 and 3 is not shown).

The right panel of Fig. 2 shows the activity of the mitral cells when a stimulus has been injected into the target neurons

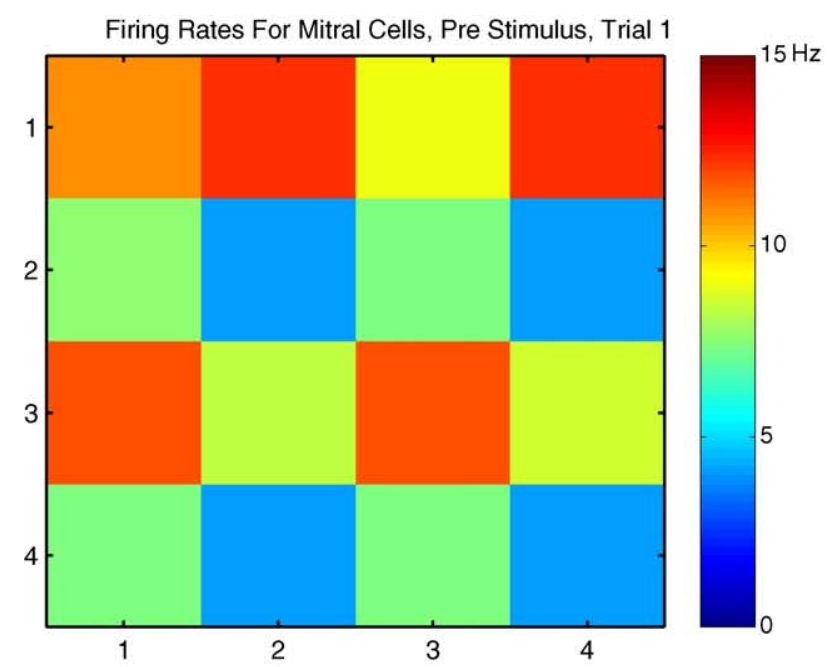

(again, mitral cell activity is similar across trials and is only shown for trial 1). From the grids it is possible to see which cells are firing fastest (red cells), the increase due to the stimulus is in the range of $30-40 \mathrm{~Hz}$. These four cells correspond to those chosen as responsive to stimulus presentation. The cells surrounding these responsive cells also show increased activity, in the range of $15-20 \mathrm{~Hz}$.

These results show that some cells increase their activity in response to stimulus presentation. The next few sections show the cells considered by MEANOVA, to have responded most strongly. If any mitral cells showed an activity of less than $5 \mathrm{~Hz}$, they were discarded and were not considered in the MEANOVA analysis.

\subsubsection{Interactions}

The interaction results show that there are no significant interactions between the changes in mitral cell activity (pre- to during-stimulus presentation) and changes in activity across trials. This can be seen in Fig. 3, where all the scores are under 1.

For 2 and $4 \mathrm{~s}$ simulations (results not shown), the scores seem to stabilise around a particular figure, depending on the number of time bins used. For $2 \mathrm{~s}$ simulation, the simulation began to stabilise at a score of 0.4 and 0.3 using 40 and 80 time bins, respectively. For $4 \mathrm{~s}$ simulation, the simulation began to stabilise at a score of 0.4 and 0.25 using 40 and 80 time bins, respectively. However, because of the short simulation durations, the reliability of this information is uncertain. Other differences in activity might be seen if the simulation was continued for longer. The results show that for longer time periods, there is a slight decrease in all the scores. For both 8 and $10 \mathrm{~s}$ simulations, scores (see Fig. 3) seem to stabilise at 0.25. As this is so for both cases, we can assume that the results are more reliable compared to 2 and $4 \mathrm{~s}$ simulations. If the simulation was continued for longer than $10 \mathrm{~s}$, we would expect similar results.

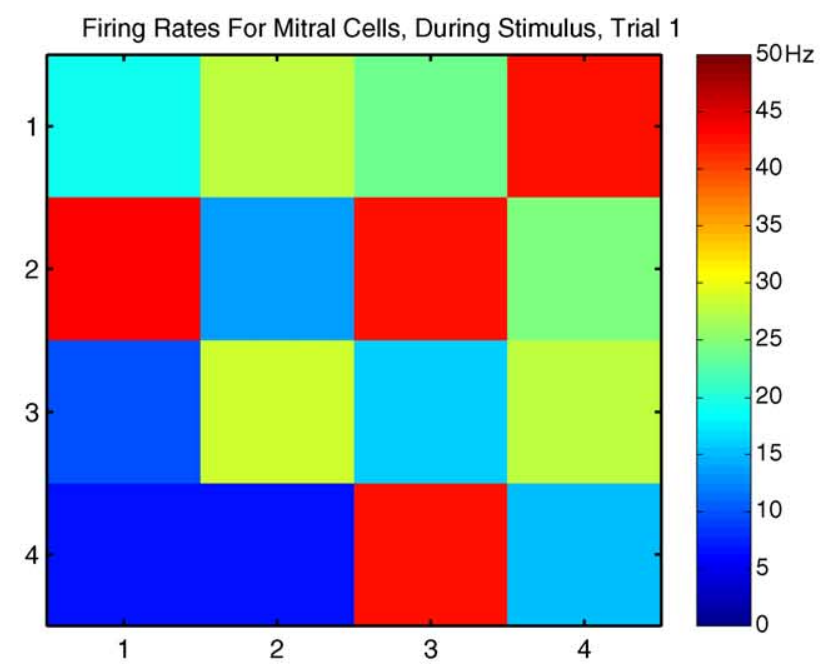

Fig. 2. Activity of the $4 \times 4$ grid of mitral cells before (left) and during (right) stimulus presentation in trial 1 . The right diagram shows an increase in the four chosen mitral cells, i.e. $04,11,13$ and 33 , refer to Table 1 (please note the difference in scales between the two diagrams). 

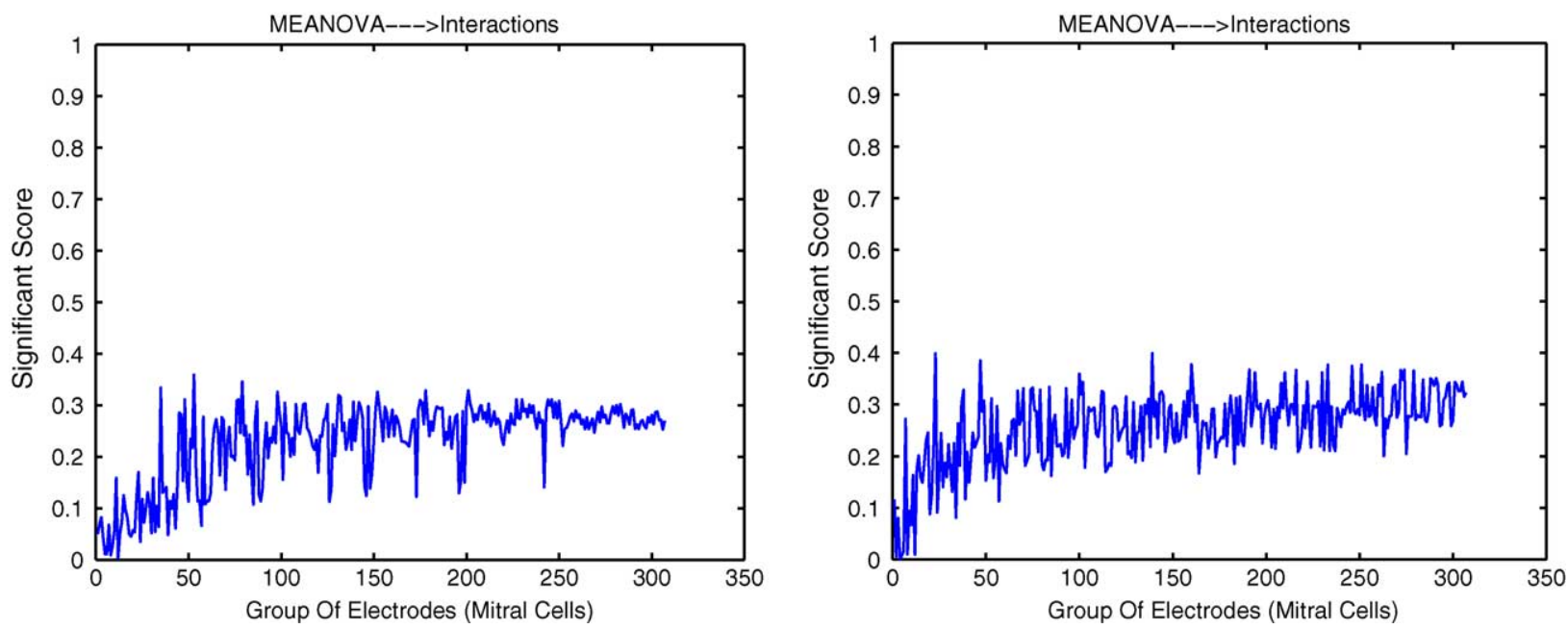

Fig. 3. Interaction results for an $8 \mathrm{~s}$ simulation with 320 time bins (left), and a $10 \mathrm{~s}$ simulation with 400 time bins (right). The individual and groups of cell scores in both graphs are shown from left to right, respectively, where the final score represents all the cells. For more details, to see which group number represents which cells, see Appendix B. These results conclude that there are no interactions, i.e. the same type of change is happening in all the tests, and that the only significant changes in the neural activity are due to either factor 1 (stimulus), factor 2 (sequential trials) or both. The fluctuations of the scores are due to the random noise injected into each cell, but this does not affect the results significantly.

The difference in scores shows that a 2 or $4 \mathrm{~s}$ simulation was not long enough to sufficiently represent the true activity of the mitral cells.

The results also show that increasing the number of time bins also alters the score. As we are looking at more points in time, we are acquiring more detail, thereby further increasing the reliability of the results.

From the results, it can be concluded that the changes in the results is due to factor 1,2 or both (as the scores for the interactions are $<1$ ). This would suggest similar change across all tests, with no interaction between the two factors. Therefore, it is possible to make a clear interpretation.

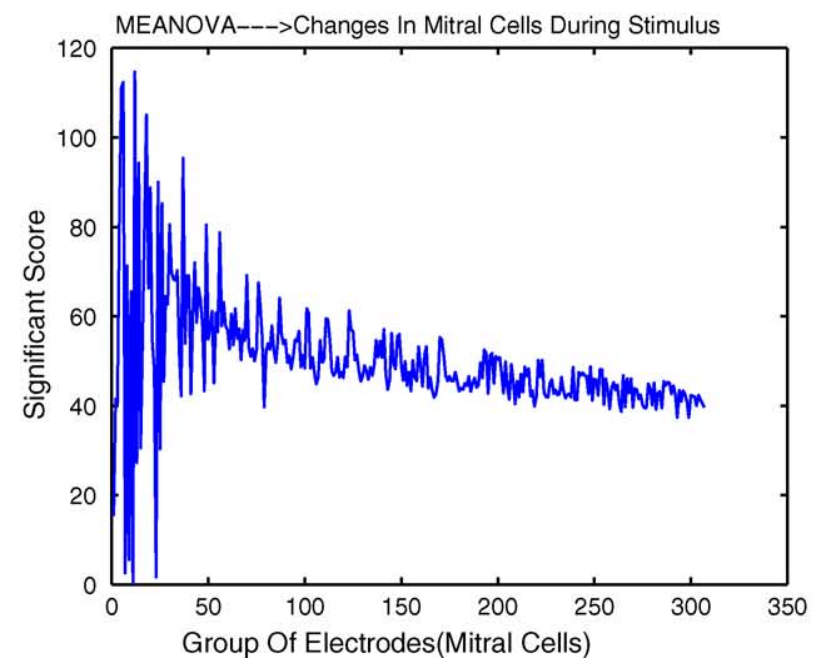

The next two sets of results show which cells in an array responded most significantly to a stimulus, and whether the responses were trial-dependent.

\subsubsection{Stimulus}

The results for a 8 and $10 \mathrm{~s}$ simulation (see Fig. 4) show that over all trials, there was a significant change in activity in individual cells and amongst groups of cells. The highest scores were found to correspond to the individual cells, which were chosen to be influenced by the stimulus. The scores for the cells surrounding those chosen had a lesser score, as they were not directly influenced by the stimulus

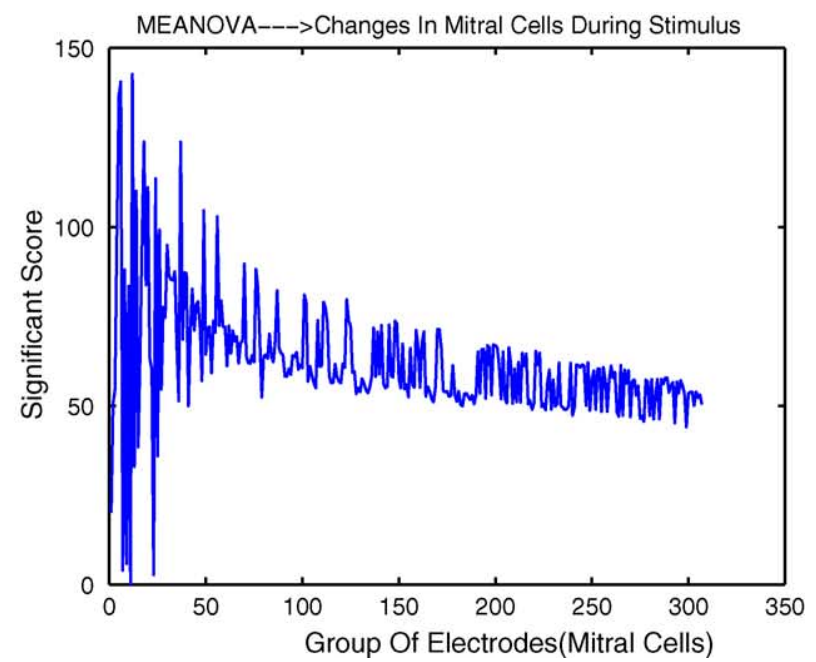

Fig. 4. MEANOVA results for an $8 \mathrm{~s}$ simulated stimulus presentation with 320 time bins (left), and a $10 \mathrm{~s}$ simulation with 400 time bins (right). The individual and groups of cell scores in both graphs are shown from left to right, respectively, where the final score represents all the cells. Appendix B shows which group number represents which cells. The results, i.e. the cells with scores $>1$ conclude that the changes in neural activity are due to factor 1 (stimulus). The exact results for the individual cells can be seen in Table 2. From the table and the graphs, it can be seen that the chosen cells, correspond to the highest scores. These results also show that the longer the simulation, the greater the score, i.e. increasing likelihood that change is significant and due to the stimulus. 
but by the connection strengths between them and the chosen cells.

The results show that the simulation results stabilise at a score of 50. This is not so in simulations of 2 and $4 \mathrm{~s}$ (results not shown), where the scores were not similar and so were less reliable.

These results show that the longer the simulation, the greater the score, i.e. increasing likelihood that change is significant.

MEANOVA clearly shows that the neurons with the most significant change are those selected to respond to the stimulus (see Table 2).

\subsubsection{Trials}

In Fig. 5, there are some slight (non-significant) changes in mitral cell activity, across trials. From this, we can conclude that a similar change in activity, in the same mitral cells, occurs in all trials in response to stimulus presentation. It shows also that significant responses do not occur in randomly varying mitral cells, but occur in a select few, those chosen to be responsive at the outset of the simulation.

Again a longer simulation (see Fig. 5), stabilises with a score in the range of $0.35-0.4$. This can be compared with a shorter duration simulation (results not shown), where the fluctuations occur in the $0.35-0.4$ range. Thus, the 2 and $4 \mathrm{~s}$ simulations would be sufficient, in this case, for reliable results.

We can conclude from the MEANOVA results, that a subset of the mitral cells responded to stimulus presentation. MEANOVA confirms that the cells selected as responsive at the outset of the simulation, are those that responded most significantly. This is supported across three trials which yielded the same results.

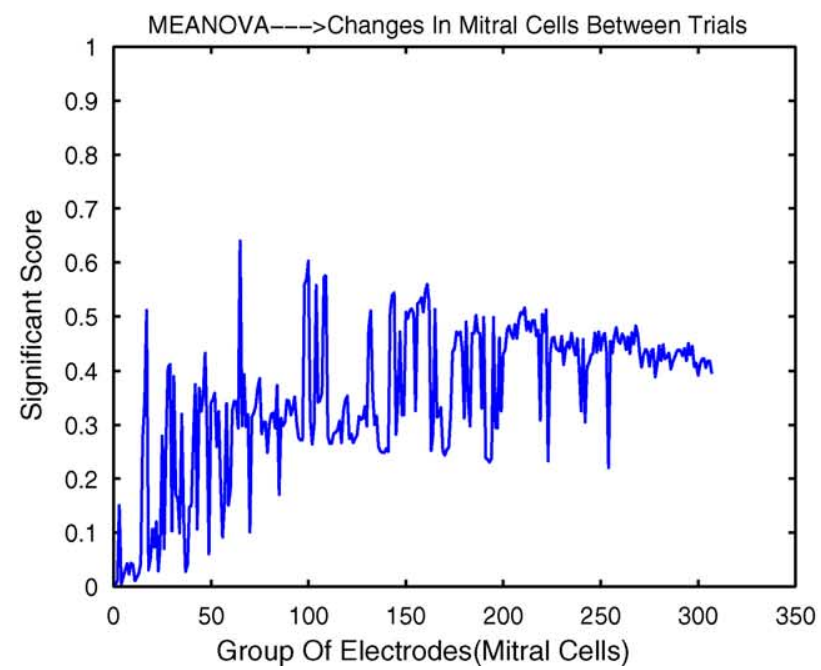

Table 2

Significant changes in activity (responses to stimulus presentation) amongst neurons in the $4 \times 4$ array, $8 \mathrm{~s}$ and $10 \mathrm{~s}$ simulation, and how significant they have changed when a stimulus is presented

\begin{tabular}{llcc}
\hline $\begin{array}{l}\text { Group } \\
\text { number }\end{array}$ & $\begin{array}{l}\text { Mitral cell } \\
\text { identity }\end{array}$ & $\begin{array}{l}\text { Score } \\
\text { (8 s simulation) }\end{array}$ & $\begin{array}{l}\text { Score } \\
(10 \mathrm{~s} \text { simulation) }\end{array}$ \\
\hline 1 & 01 & 15.34 & 19.9811 \\
2 & 02 & 41.4529 & 50.6718 \\
3 & 03 & 40.5671 & 54.5224 \\
4 & 04 & 83.056 & 100.3722 \\
5 & 11 & 111.1561 & 136.8276 \\
6 & 13 & 112.3447 & 140.5853 \\
7 & 21 & 2.5754 & 3.9188 \\
8 & 22 & 71.274 & 88.0452 \\
9 & 23 & 5.5029 & 5.8454 \\
10 & 24 & 65.7228 & 83.7931 \\
11 & 31 & 0.3358 & 0.026792 \\
12 & 33 & 114.6635 & 142.7194 \\
\hline
\end{tabular}

It is possible to see that the chosen cells (shown in italic) have the highest scores. Due to the excitatory connections between the chosen and the surrounding cells, the majority of the cells have had an increase due to the stimulus, but the change has not been as big compared to the chosen ones. Mitral cell ID 31 is the only one not connected to any of the chosen cells, so the score concludes that it has slightly changed in activity, but not due to the stimulus, i.e. the score being $<1$, but to other factors, in this case, the random noise added to the cell.

\subsubsection{Group plotting}

In order to easily assess the impact of stimulus presentation on each cell and its surroundings, we can plot the MEANOVA results for each cell and all groups containing that cell.

For example, in Fig. 6 (right), the significant score is plotted for all groups containing cell 31 . Hence for cell 31 alone, its significant score is very low $(<1)$. This implies that the cell does not change its activity in response to

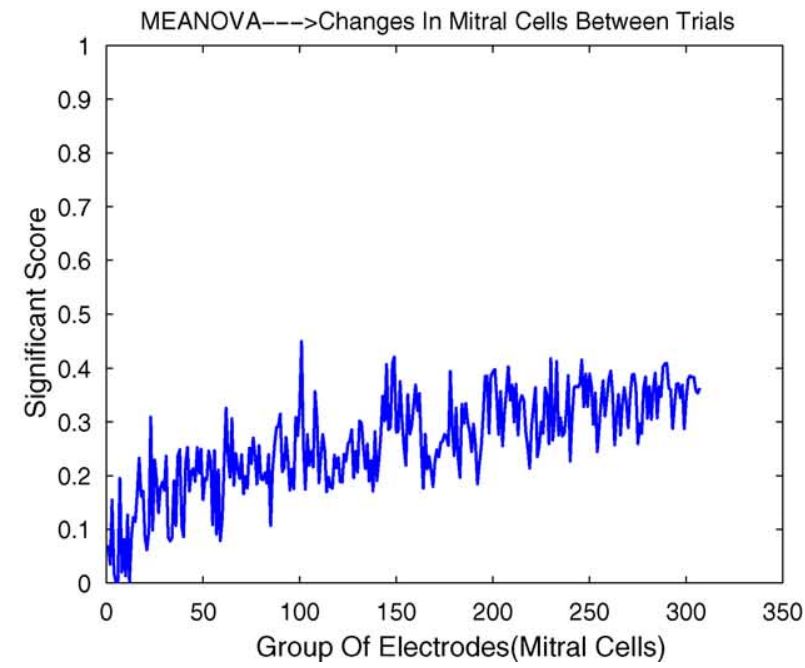

Fig. 5. MEANOVA results for different trials with simulated stimulus presentation of $8 \mathrm{~s}$ with 320 time window (left), and a $10 \mathrm{~s}$ simulation with 400 time bins (right). The individual and groups of cell scores in both graphs are shown from left to right, respectively, where the final score represents all the cells. For more details, to see which group number represents which cells, see Appendix B. These results, i.e. the scores are all $<1$ conclude that there are no changes in neural activity due to factor 2 (the sequential trials). These results also show that the longer the simulation, the lesser the score, i.e. increasing likelihood that no change has occurred due to there being repeated presentations of the stimulus (sequential trials). The results also show that there was some change between trials, but nothing significant. This is due to the random noise injected into all of the cells. 

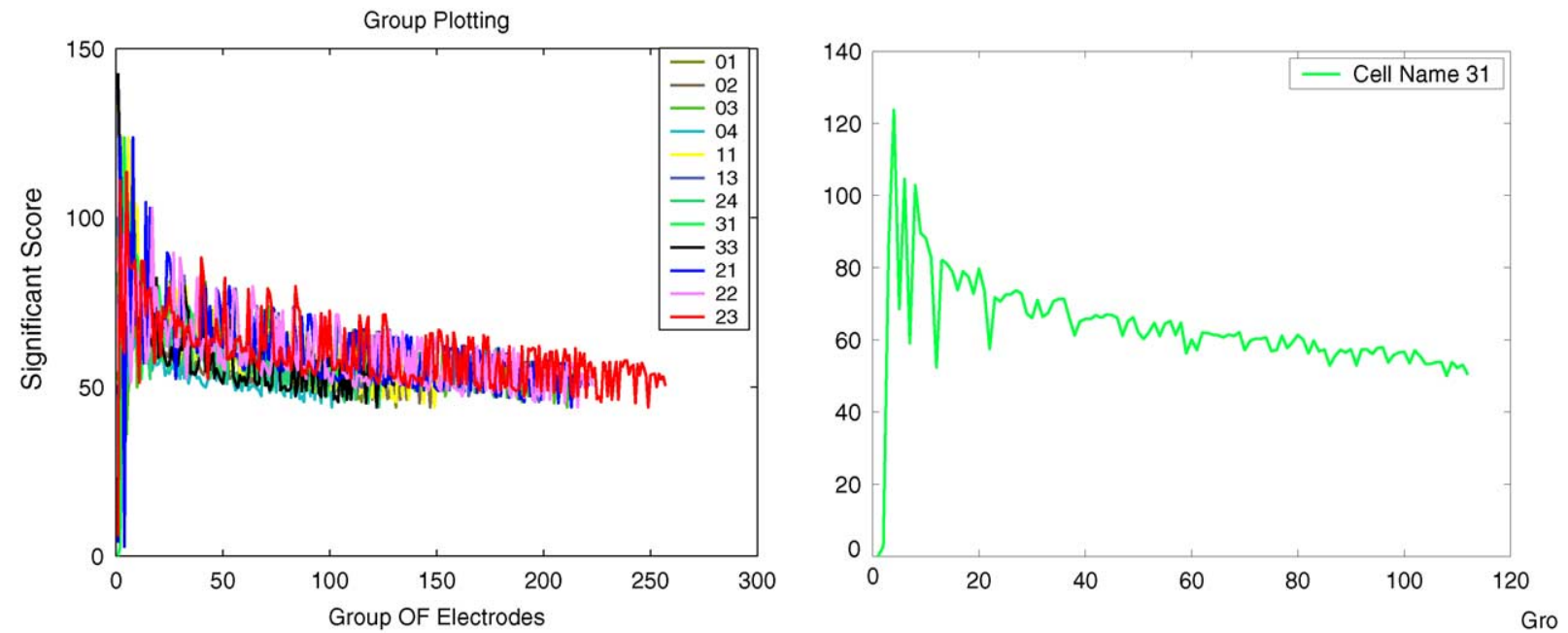

Fig. 6. Group plot of MEANOVA results. Left: significant score for all 12 cells and their associated groups. Right, significant score for cell 31 . Hence, the first point corresponds to 31 , the second point to $23=(21,31)$, the third point to $37=(11,21,31)$, etc. (see Appendix B).

stimulus presentation. However, when considered together with cell 21 , its significant score is 2.5 , i.e. there is a significant response to stimulus presentation for the group $(21,31)$.

\subsection{Results generated from model for second experiment}

This experiment simulates activity across a $9 \times 9$ grid of mitral cells. It is implemented to demonstrate that if there is a small stimulus-related activity change in a few of the 81 mitral cells, MEANOVA can detect which of those cells respond most significantly. The presented stimulus has been reduced to deliver a maximum of $20 \mathrm{nA}$ to the network.

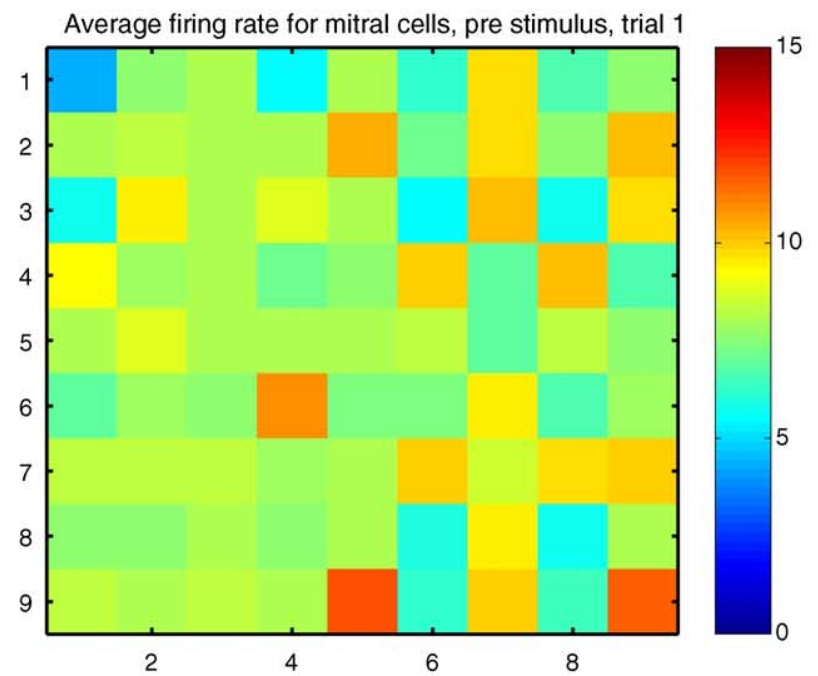

- The first test shows the results when many of the cells were selected as responsive and strongly influencing their neighbouring cells.

- The second shows the results when only four mitral cells were responsive with little influence over surrounding cells.

- In the third and final test, only a single mitral cell was responsive.

The maximum number of groups permitted was 50,000, a reasonable ceiling, as by this point, the groups would be stabilising around a particular score. All tests have three $10 \mathrm{~s}$ simulations (i.e. three trials) and 40 windows/s. The inputs to each cell, before stimulus onset, were fixed across all tests and trials (see Fig. 7 left for trial 1; trials 2 and 3 have very

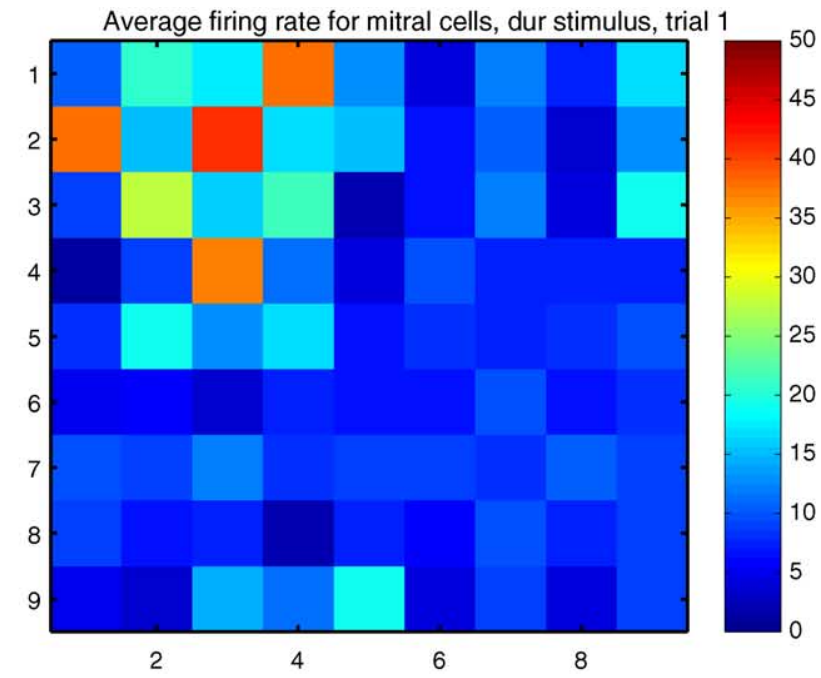

Fig. 7. Activity of all mitral cells across the $9 \times 9$ cell grid before (left), and during (right) stimulus presentation in trial 1 when four cells were selected as responsive and strongly influenced the activity of their neighbouring cells. The four chosen mitral cells are $04,11,13$ and 33 , refer to Table 3 . Note the difference in firing rates due to the decrease in the strength of the stimulus, compared to the $4 \times 4$ grid in Fig. 2 (please note the difference in scales between the two diagrams). 
Table 3

Target neurons are shown in italic

\begin{tabular}{lllllllll}
\hline 01 & 02 & 03 & 04 & 05 & 06 & 07 & 08 & 09 \\
11 & 12 & 13 & 14 & 15 & 16 & 17 & 18 & 19 \\
21 & 22 & 23 & 24 & 25 & 26 & 27 & 28 & 29 \\
31 & 32 & 33 & 34 & 35 & 36 & 37 & 38 & 39 \\
41 & 42 & 43 & 44 & 45 & 46 & 47 & 48 & 49 \\
51 & 52 & 53 & 54 & 55 & 56 & 57 & 58 & 59 \\
61 & 62 & 63 & 64 & 65 & 66 & 67 & 68 & 69 \\
71 & 72 & 73 & 74 & 75 & 76 & 77 & 78 & 79 \\
81 & 82 & 83 & 84 & 85 & 86 & 87 & 88 & 89 \\
\hline
\end{tabular}

similar activity and are not shown). The grid layout and cells selected as responsive were also fixed across all tests and are shown in Table 3.

\subsubsection{Test 1}

The interactions and the scores for the changes between trials were all under 1 (results not shown).

The results shown in Fig. 7 (right) show mitral cell activity for trial 1, during-stimulus presentation when four mitral cells were selected as responsive and strongly influenced the activity of their neighbouring cells. The neighbouring cells also reciprocally affected the chosen cells (trials 2 and 3 show a similar pattern of mitral cell activity, results not shown). The cells selected as responsive showed the highest score, compared to their neighbours, which showed a lower score, due to the stimulus not directly affecting them (Fig. 8). The rest of cells, which were not laterally connected to the chosen cells, had lower scores due to the stimulus not affecting them. The scores gained for the chosen and surrounding cells

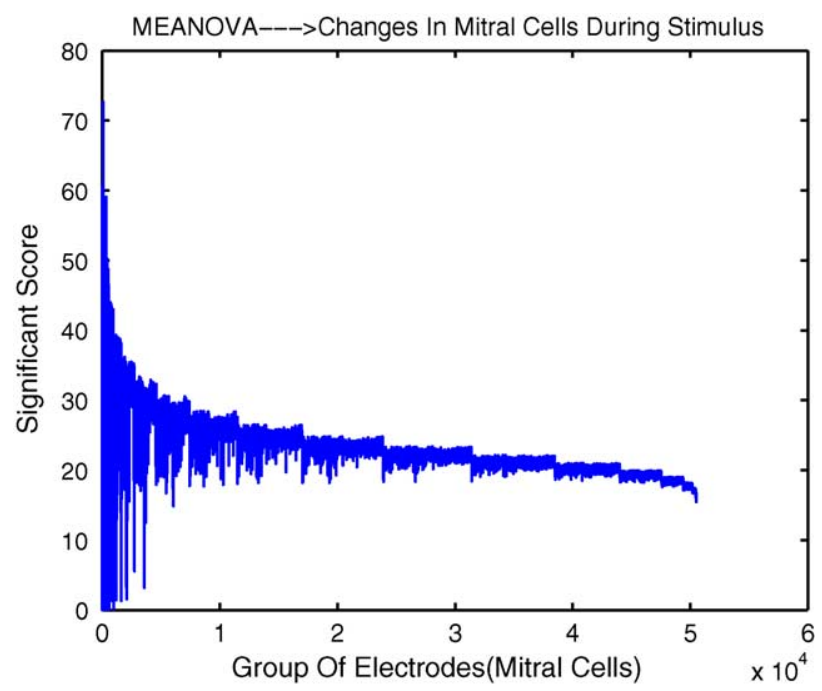

Fig. 8. MEANOVA results for pre- and during-stimulus activity to determine whether any of the mitral cells responded significantly to stimulus presentation. The individual and groups of cell scores in the right graph are shown from left to right respectively, where the final score represents all the cells. These results, i.e. the cells with scores $>1$ conclude that the changes in neural activity are due to factor 1 (stimulus). There are many more scores below 1 , as about half of the cells are not changing activity due to the stimulus. The scores are smaller compared to the scores in Fig. 4, as there was a decrease in stimulus.
Table 4

This table shows the scores for the four chosen cells in all three tests implemented

\begin{tabular}{rllll}
\hline $\begin{array}{l}\text { Group } \\
\text { number }\end{array}$ & $\begin{array}{l}\text { Mitral cell } \\
\text { identity }\end{array}$ & Score test 1 & Score test 2 & Score test 3 \\
\hline 1 & 04 & 60.525 & 54.7198 & 0.00226 \\
6 & 11 & 52.859 & 44.4104 & 0.0039322 \\
8 & 13 & 53.582 & 47.8202 & 0.070774 \\
22 & 33 & 51.331 & 46.5248 & 3.7146 \\
\hline
\end{tabular}

The table shows that the neurons, which were selected to be responsive to a stimulus, achieved the highest score, this can be seen in the tests 1 and 2 results (score test 1 and score test 2 columns, respectively). The scores in test 1 are slightly higher than in test 2 , as the surrounding cells were not effected by the chosen cells, the surrounding cells were firing less, than in test 1 , which contributed less to the chosen cells, resulting in a lower score. The test 3 scores show that as there was only one cell (Cell 33) affected by the stimulus as it gained the only score $>1$. The rest of the cells scored below 1. There was a decrease in the score for Cell 33 in test 3 , compared to the other 2 tests, due to the decrease in stimulus, resulting in a corresponding decrease in the strength of the response.

were not as high as the $4 \times 4$ grid results, due to the decrease in stimulus strength. Table 4 shows the chosen cells' scores.

The results signify that factor 1 has had significant affects in changing the activity of some of the neurons.

\subsubsection{Test 2}

The next set of results depicted in Fig. 9 show the activity of the mitral cells, during a stimulus presentation, when four mitral cells were selected as responsive and did not influence the activity of their neighbouring cells. The connections from the surrounding cells to the chosen ones had excitatory connections which also affected the chosen cells.

Fig. 9 (left) shows the activity of the mitral cells duringstimulus presentation in trial 1 (trials 2 and 3 show similar mitral cell activity and are not shown). In Fig 9 (right), it can be seen that, because of the change in activity of only four of the neurons, the significant scores for most groups is lower than test 1 . The groups with the highest scores, include at least one of the four cells selected as responsive (see Table 4; score test 2 section for chosen cell scores). Most of the cells had a score $<1$ which showed that a slight change in activity had occurred but was not due to the stimulus but due to the random noise.

Again, for the interactions and for the changes between trials, all scores were under 1 (not shown).

\subsubsection{Test 3}

Finally, in test 3 , only one neuron is selected as responsive, and its response to a stimulus input of $0.1 \mathrm{nA}$ is only slightly significant. The selected cell increased its activity to $4 \mathrm{~Hz}$ (cell 33 in this case; see Fig. 10 and Table 4).

The interactions and the changes between the trials all scored $<1$ (not shown).

It can be seen (Fig. 10, right) that the highest score in the graph corresponds to the single responsive neuron. Also shown (Fig. 10, left) is the mitral cell activity in trial 1 when 

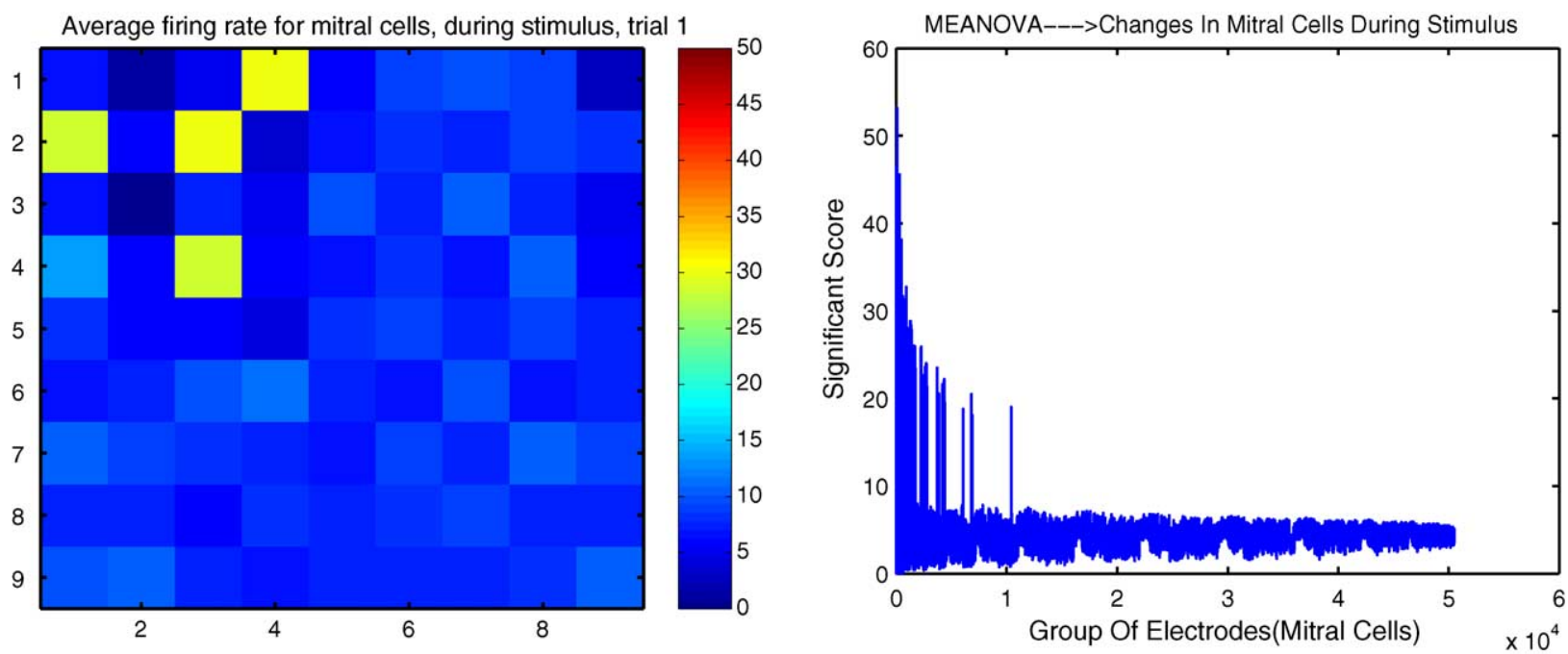

Fig. 9. The graph on the left shows the activity of all mitral cells across the $9 \times 9$ cell grid during a stimulus presentation for one trial. The results from the grid show that four cells were selected as responsive and had no influence over the activity of their neighbouring cells. This is confirmed in the right graph where the highest scores correspond to the selected cells. The individual and groups of cell scores in the right graph are shown from left to right, respectively, where the final score represents all the cells. These results, i.e. the four cells with scores $>1$, conclude that the changes in neural activity are due to factor 1 (stimulus). The rest of the cells were not changed so have scores $<1$. Overall when examining more and more of the cells together, the significant score drops compared to Fig. 8 , this is because many more cells had no change in activity.

only one cell responded to stimulus presentation (trials 2 and 3 show similar mitral cell activity and are not shown). As more cells are incorporated into the analysis, the scores decrease. Thus, with only one significantly responsive neuron, analysis of the entire group of neurons reveals no significant change in activity, bearing out the assumption that brute-force application of MANOVA buries sparse information. It also demonstrates that a slight increase in activity can be found and linked to a presented stimulus.

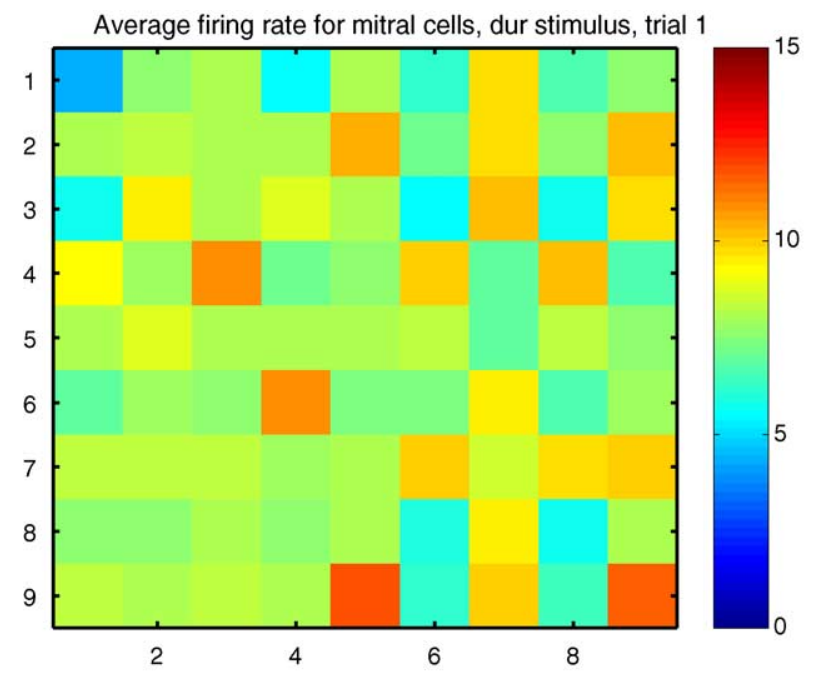

The simulation results have shown that the use of this method can be important in the use of practical applications. This is due to the method being able to find one or more neurons, which have responded to either a high or low level of stimulus (hot-spots) and that the change was due to the stimulus being presented and not by chance. These factors would show how biologically important the findings are, when applied to experimental data. This is discussed in the next section.

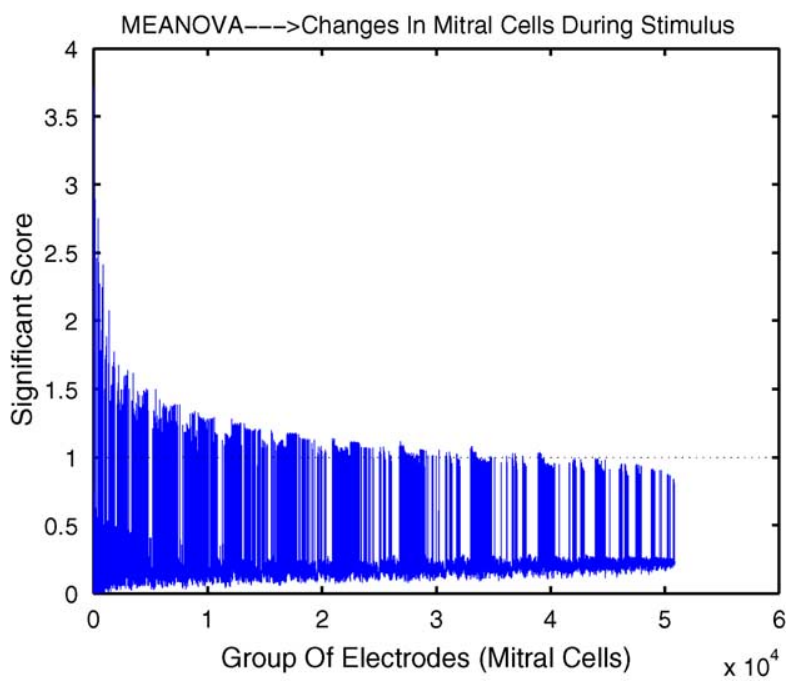

Fig. 10. The graph on the left shows the activity of all mitral cells across the $9 \times 9$ cell grid during a stimulus presentation for one trial. The results from the grid show that a single cell was selected as responsive and had no influence over the activity of its neighbouring cells. This is confirmed in the right graph where the highest score corresponded to the only selected cell. The individual and groups of cell scores in the right graph are shown from left to right, respectively, where the final score represents all the cells. From these results, it can be concluded that the changes in activity of the single responsive cell (the only cell with a score >1) was due to factor 1 (stimulus), but achieved a lower score compared to the score of test 2 (shown in Table 4), due to a decrease in stimulus input. The rest of the cells were not changed so have scores < 1 . Overall when looking at more and more of the cells together, the significant score dropped $<1$ compared to Figs. 8 and 9, this is because many more cells had no change in activity. 


\section{Application to recordings from rat olfactory bulb}

\subsection{The olfactory bulb}

The OB is probably the simplest system to be explored in addressing the issue presented at the start of this paper (Kendrick et al., 1997; Uchida and Mainen, 2003). In the mammalian olfactory system, much encoding of odour information takes place in the OB (Desmaisons et al., 1999; Bracci et al., 2003; Debarbieux et al., 2003; Egger et al., 2003; Neville and Haberly, 2003; Pinato and Midtgaard, 2003). Olfactory receptor neurons project to glomeruli in the OB, where they synapse with efferent mitral cells. The quality of a particular odour is believed to be encoded in the specific combination of glomeruli activated by that stimulus.

By using MEANOVA to analyse neuronal activity captured using an MEA in the olfactory bulb of the anaesthetised rat, we hope to find an area (hot-spot) or areas of neurons which respond significantly to a stimulus (odour). Questions that can be answered from the analysis are whether the stimulus is encoded spatially in small groups, i.e. in few neurons, or whether it is encoded in a much larger area where many neurons are being used for the encoding process. It will also be possible to see, due to the sequential trials, if the neurons respond to a repeatedly presented stimulus and what affects it has on the neural activity. Examining the results created from this analysis will give a quick and clear interpretation of the activity of the olfactory bulb in the MEA recorded area when a stimulus is present.
Before a MEANOVA analysis can be performed on the data from the rat olfactory bulb, the MEA data must first be sorted. Each electrode samples a continuous neural trace, but spiking is detected, and activity is captured when the voltage increases above a certain threshold. The spikes captured from each electrode are grouped according to their waveform characteristics (more details on this process will be presented in a forthcoming paper). As the recordings are affected by extraneous noise, we try to group the waveforms which have similar characteristics. This process results in the number $(P)$ of individual neurons being identified and associated to each electrode, where each neuron has a number of action potentials (spikes) associated with them.

As discussed in the simulation section, the recording time and the width of the time bins are problems when analysing neural activity. These need to be chosen wisely, so that we can obtain and interpret accurate scores from the meanova process. Details of these parameters and the experiment are as follows.

Under urethane anaesthesia (25 urethane i.p., $1.5 \mathrm{~g} / \mathrm{kg}$ ), rats were fixed in a stereotaxic frame and an MEA (e.g. $5 \times 6$, 30 electrodes) was positioned laterally in the OB. Recordings of activity from individual neurons (spikes) and local field potentials were made from each electrode. Humidified air was supplied to the rat via a mask over the nose. Shortly (30 s) before delivery of an odour stimulus, the air to the rat was switched to dry air. Odours, carried as saturated vapours in nitrogen gas (odourless), were mixed in various concentrations with dry air and were delivered (10 s) to the rat via

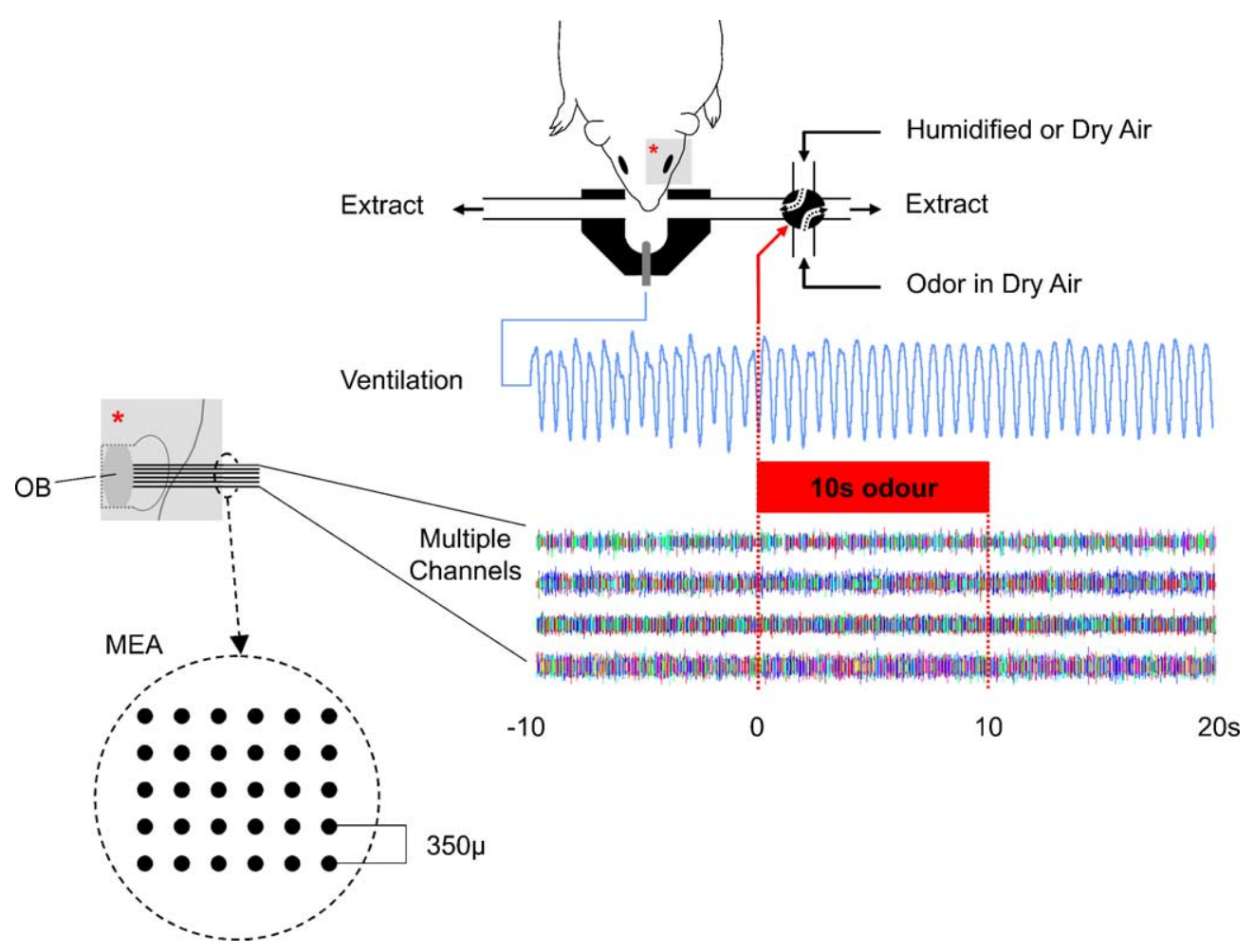

Fig. 11. A schematic plot of the experimental procedure. Selected channels are shown. For each channel, spikes from $<11$, neurons were discriminated. 
the mask; three presentations were made of each odour at each concentration, with an inter-trial interval of $\geq 3$ min. Breathing was monitored and onset of odour delivery was precisely timed to mid-exhalation (see Fig. 11). Thus, delivery of the odour to the nasal epithelia would have commenced from the start of the subsequent inhalation (Nicol et al., 2003).

In our analysis below, we take $t=200 \mathrm{~ms}$ and there are $M=T / t=25$ windows for each cell. We also test all results for $t=400 \mathrm{~ms}$ and the results are presented in http://www.sussex.ac.uk/Users/pmh20. It is noted that there are no significant changes between $t=200$ and $400 \mathrm{~ms}$.

\subsection{Results}

In accordance with MEANOVA, we can compare neuronal activity with many factors: trials, odours, concentration, etc., as developed in our software. However, here we exclusively consider two factors: factor 1 , pre- and during stimulus, and factor 2, different trials.
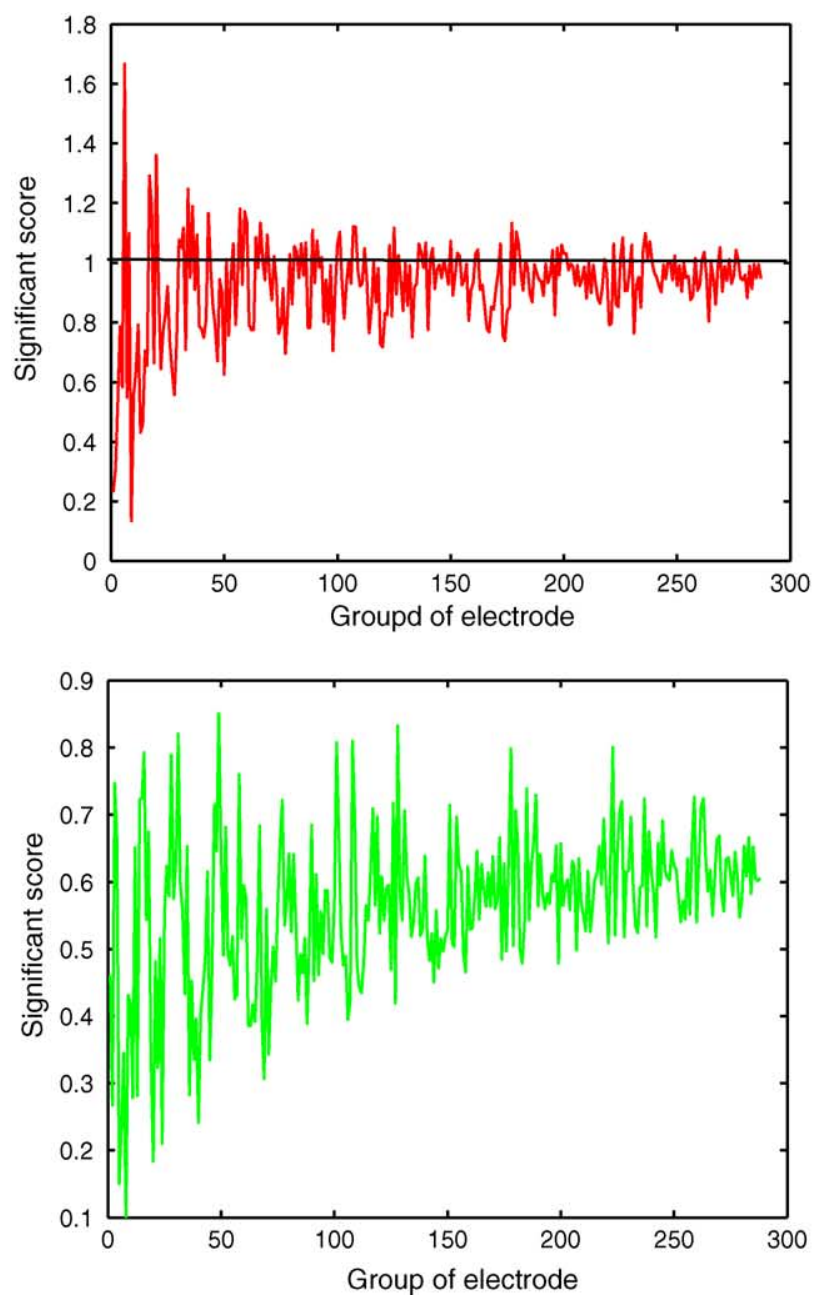

\subsubsection{Representations}

The first difficulty we encounter is the representation problem. Assume we have a two-dimensional array of electrodes enumerated according to $\{i, i=1, \ldots, Q\}=\mathcal{Q}$.

Let us first estimate whether this is possible for an array of electrodes. Assume that neuronal activity has been sampled from every electrode in the array. Each electrode in the array has four neighbours, and hence, in total we have

Total number of groups $>3^{\sqrt{Q}}$

Therefore, the number of groups we have to calculate increases exponentially with the total number of electrodes.

In our recordings, we find that electrodes which sample neuronal activity are usually sparse, but the number may nonetheless be high. In the next section of MEANOVA analysis, we simply map a group of electrodes to a single digital number, as shown in Appendix A. The total number of neighbouring electrodes is 286 , comparable with $3^{\sqrt{30}}$.
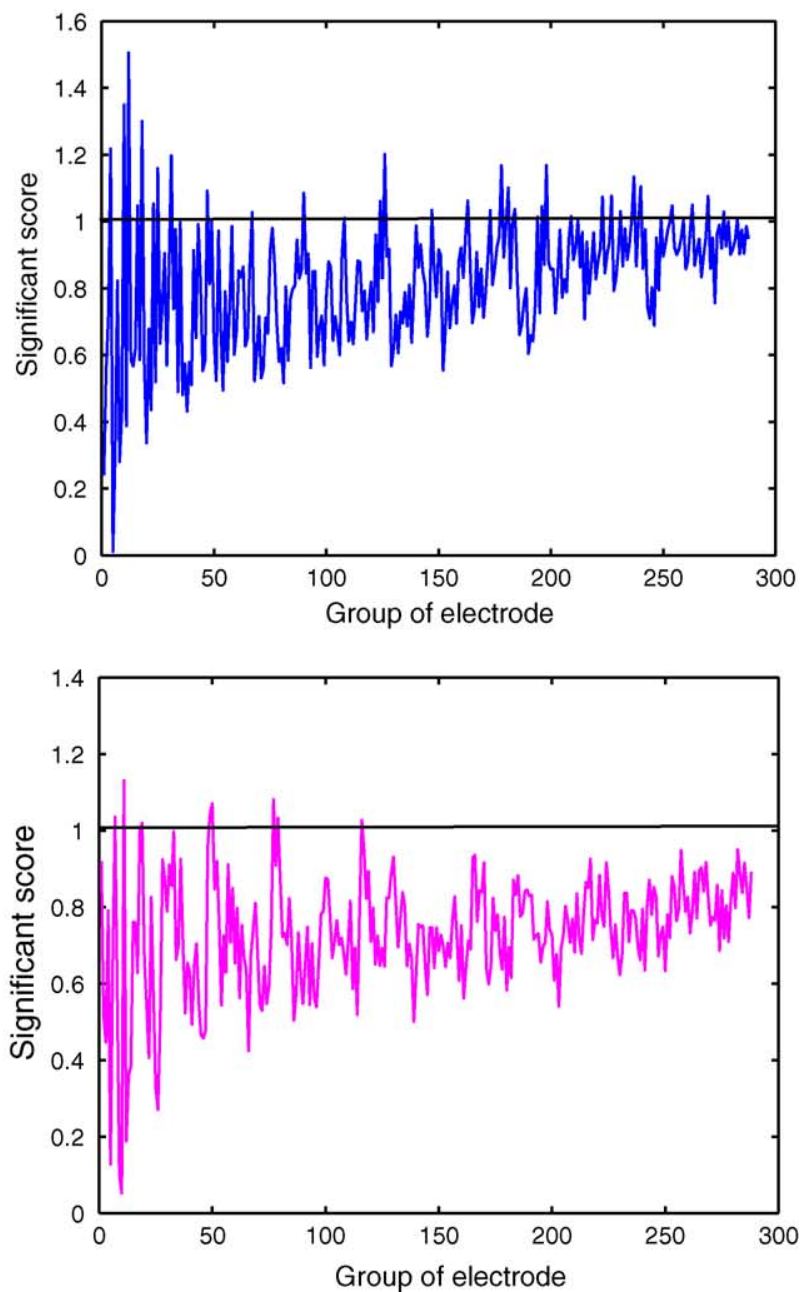

Fig. 12. MEANOVA results for interactions for concentrations 1, 2 (upper panel, from left to right), 3 and 4 (bottom panel, from left to right). The individual and groups of cell scores in each graph are shown from left to right, respectively, where the final score represents all the electrodes analysed together. For more details, to see which group number represents which electrodes, see Appendix A. 


\subsubsection{Interactions}

As we mentioned earlier, in order to assess the changes due to each factor, we have to look at the effect of interactions first.

The interaction results are examined to see if either of the factors have a clear affect on the neuronal activity. Any interactions, i.e. scores $>1$, would show that other factors are present which are affecting the neuronal activity. This would show that the variances in the data are not accounted for by either factor 1 or 2 . If this is the case, it would be harder to interpret if factor 1 or 2 are having any affect. If this was the case there would be no cause to investigate the two factors separately as we would not be able to get a clear interpretation.

If there are no interations, i.e. scores $<1$, then we can conclude that any changes made to neuronal activity was directly from either factor 1 or 2 ; in this case, the residual variance would be small over all levels of the factor. To investigate this further, the next two sections investigate the affect of individual factors on the neuronal behaviour.
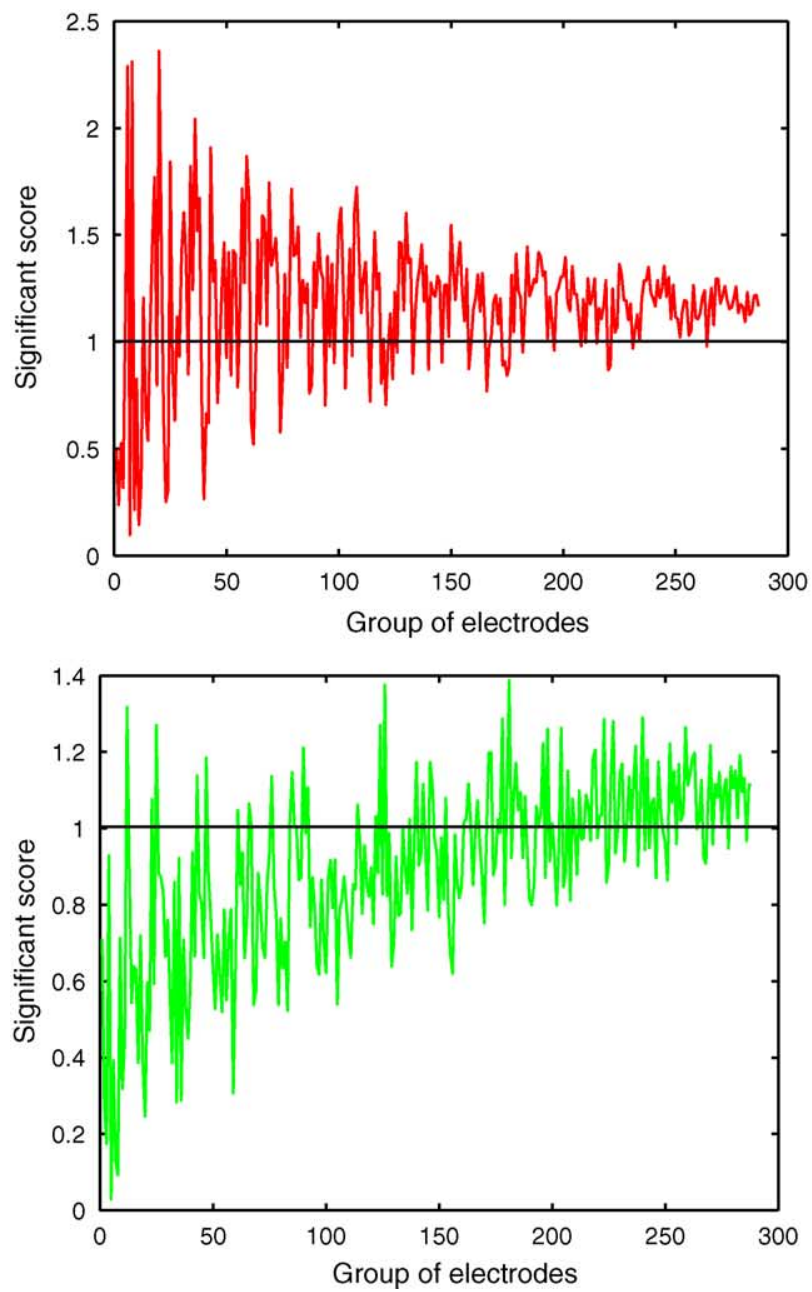

In Fig. 12, the significant scores for different concentrations are shown. We see that, in general, the interactions are small, with a score smaller than 1 . However, for concentrations 1, 2 and 4, some scores are greater than 1, indicating that we have to be careful when we interpret these results. Each of the graphs show individual electrode scores, combinations of the electrodes analysed together (i.e. to analyse a bigger area), and finally, the last score would be related to every electrode, which would show an overall score for the entire area recorded by the MEA, the individual and group scores are shown from left to right, respectively.

For concentration 3 , it is perfectly safe to look at the effect of each factor. When the interaction between two factors is bigger than one, we then have to resort to oneway MEANOVA, also included in our software. Since the two-way MEANOVA is more complicated, we confine ourselves to the two-way MEANOVA in the current paper.
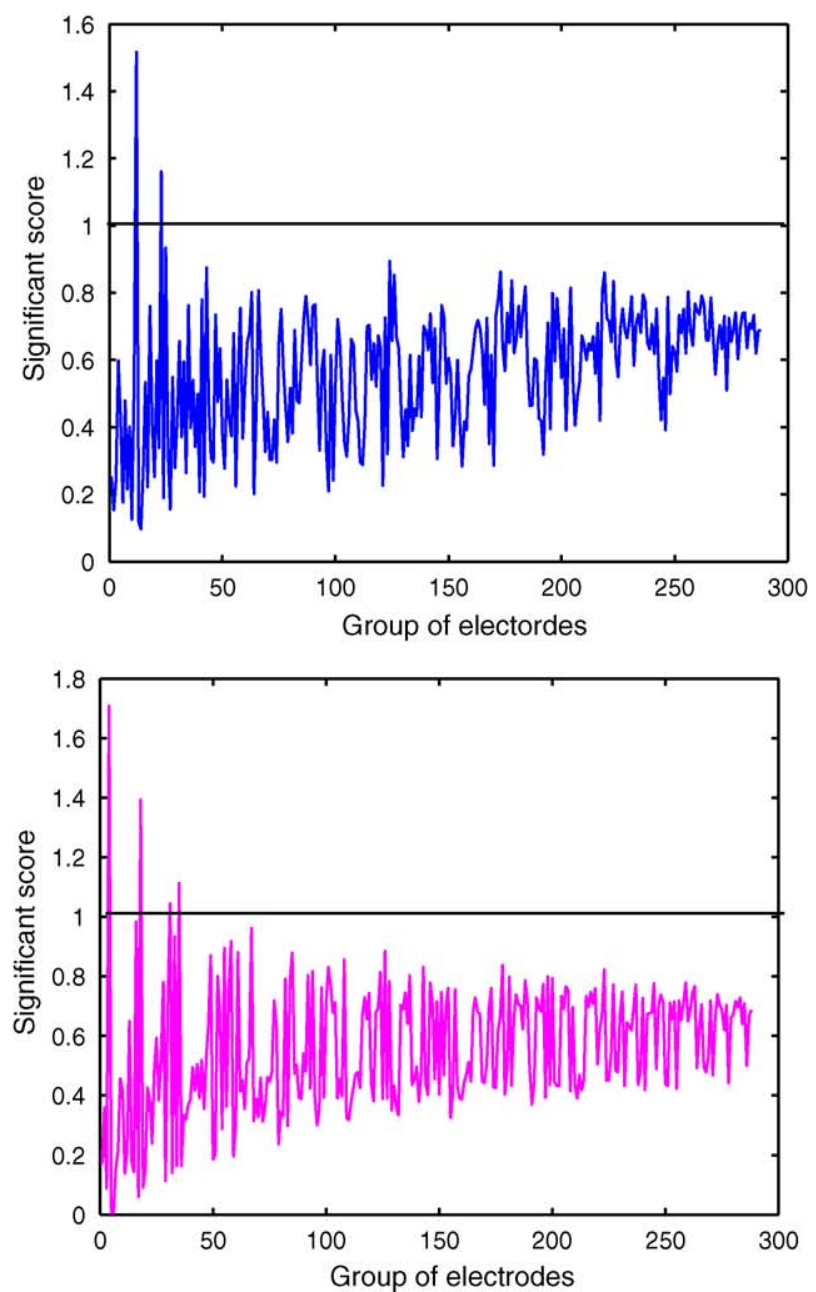

Fig. 13. MEANOVA results for factor 1, pre- and during stimulus for concentrations 1, 2 (upper panel, from left to right), 3 and 4 (bottom panel, from left to right). The individual and groups of cell scores in each graph are shown from left to right, respectively, where the final score represents all the electrodes. For more details, to see which group number represents which electrodes, see Appendix A. The results show different neural responses in different parts of the recorded area, when different concentrations of the odour are presented. The graphs representing concentrations 1 and 3 show some areas of mitral cells respond more significantly than others when presented with a stimulus. 


\subsubsection{Stimulus}

First we intend to test whether there are significant changes between pre- and during-stimulus activity (see Fig. 13). In general, we can assess that there are some significant changes, i.e. some of the areas recorded by the MEA achieved scores $>1$ for the first and the third concentrations. Some of the scores are higher than others, which means that some of the areas of the olfactory bulb are responding more actively than others.

From this analysis, we can state that not all of the recorded area responds to a stimulus, but a proportion does. The second and the fourth concentrations have shown that although few groups of electrodes show significant changes, we note that the fluctuations are large and possibly random.

\subsubsection{Trials}

The results in the previous subsections are quite curious and it is natural to consider what happens. Now we turn our attention to the effects of different trials.
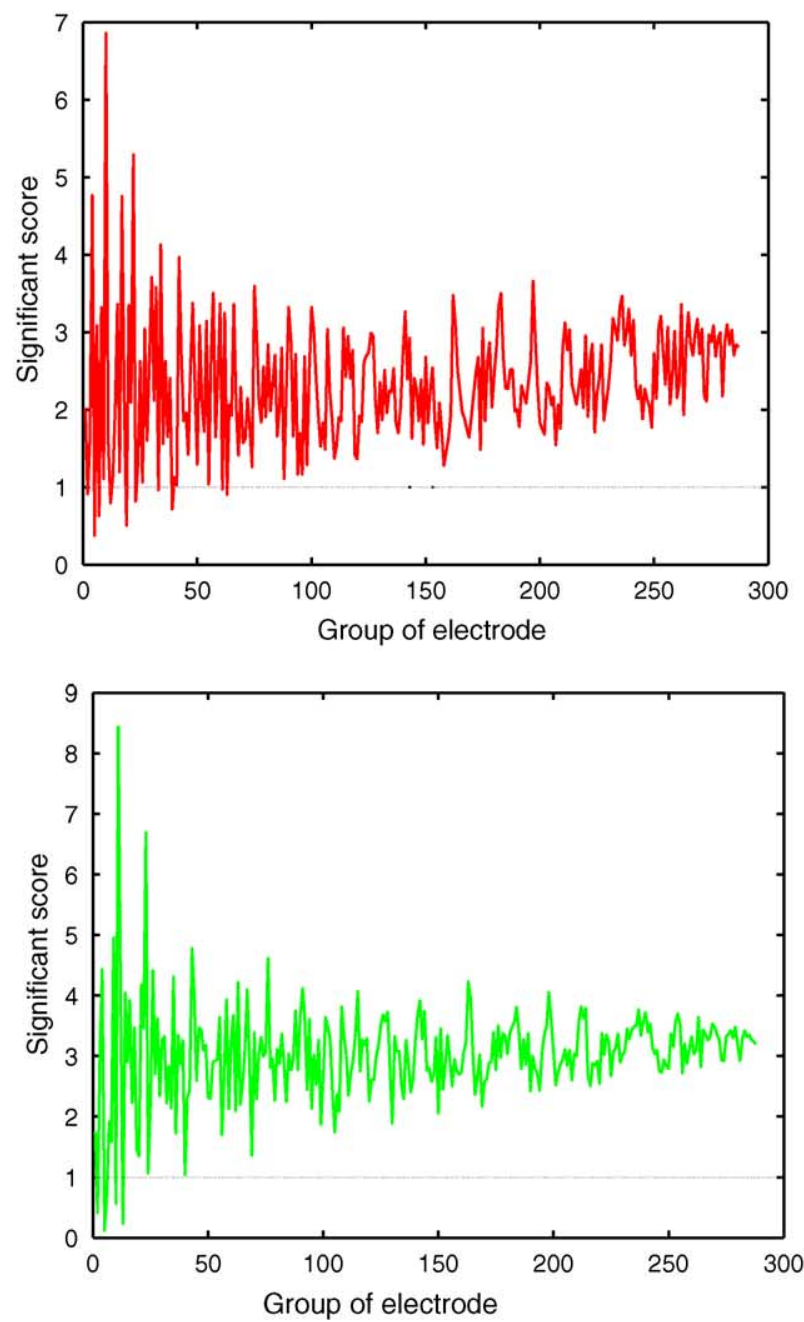

In Fig. 14, it is interesting to see that the trial effector is quite significant, scoring much higher than the score for the comparison of pre- and during-stimulus activity. This change in activity has been shown over the whole area of olfactory bulb recorded by the MEA, due to all of the significant scores being $>1$. Remembering that we performed the recordings in a sequential way (see before), the results imply that the effect of habituation becomes dominant within a $5 \mathrm{~s}$ period of odour delivery.

In order to check our results, further tests (KolmogorovSmirnov test, also included in our software) were performed on single neuron activity. For the same data, it is found that on average, there are 22 neurons which show significant changes between different trials (trial 1 against trial 2, trial 1 against trial 3 and trial 2 against trial 3) pre-stimulus, but there are only 16 neurons that change significantly between pre- and during stimulus (over the three trials). Therefore, single cell results are in agreement with our MEANOVA results.
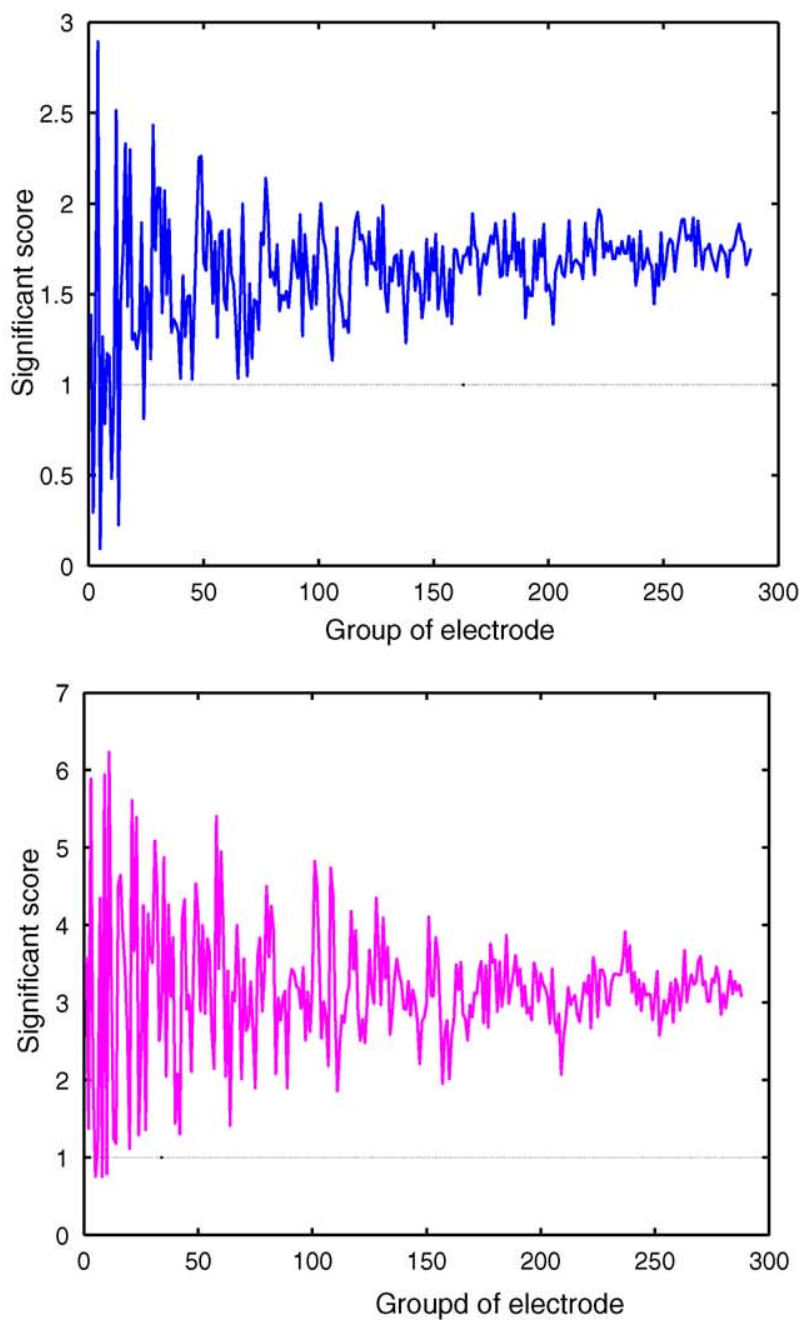

Fig. 14. MEANOVA results for trial factor with concentrations 1, 2 (upper panel, from left to right), 3 and 4 (bottom panel, from left to right). The individual and groups of cell scores in each graph are shown from left to right, respectively, where the final score represents all the electrodes analysed together. For more details, to see which group number represents which electrodes, see Appendix A. The results show that repeating a stimulus to the olfactory bulb has caused a change in the mitral cells throughout the whole area recorded by the MEA, with some parts of the area responding more actively than others. 


\subsubsection{Fluctuations}

It is interesting to note that the magnitudes of fluctuation of the significant score decrease as $|\mathcal{S}|$ increases. In other words, with a large value for $|\mathcal{S}|$, the obtained results are more reliable. Hence, it is natural to ask whether the observed phenomena in the previous subsections are general, or depend on our specific data. In Appendix C, we show theoretically that this should be the case.

\section{Discussion}

We have presented a novel method for applying MANOVA to the analysis of electrophysiological data using a multielectrode array. By applying this method (MEANOVA) to both biological and simulated data, we conclude that this novel approach is useful for detecting the areas within the array that are most significantly responsive to external stimulation.

The score details the difference in the activity of a cluster of neurons, and if compared with the other scores, provides an insight into whether the level of change throughout the area recorded by the MEA is different. In addition to this, the score also provides a confidence level that the change in neural activity was due to one of the factors under investigation and not just by chance.

From the perspective of methodology, we can say that MEANOVA is a valuable extension of MANOVA. As the number of electrodes increase, the variation becomes stable, i.e. a more reliable result is obtained. The MEA employed in acquiring the biological data presented here was quite small; neuronal activity was recorded from 11 electrodes (see Appendix A). However, even with relatviely small MEA, we achieve near stablility in the results.

Analysis of simulated data also demonstrated that MEANOVA yields useful results irrespective of the size of array used. Whilst only 11 electrodes were used to sample the biological data, the simulation demonstrates that MEANOVA is equally effective with a much larger number of electrodes. Furthermore, we establish that the number of observations (time bins) and duration of sampling, are both important factors. For example, application of MEANOVA to a short period of recording, can yield inaccurate information about the true character of the data.

It should be emphasised that MEANOVA can detect one or several cells responding strongly to stimulus presentation even when this responsiveness is embedded within a large sample of unresponsive cells. MANOVA would hide such sparse yet highly significant change. Hence when recording from a large array of electrodes, MEANOVA will play a vital role in highlighting hot-spots of activity across the recording area.

Finally, we want to emphasize that although we exclusively discuss our approach for dealing MEA data, this technique is readily applicable to many other types of data including gene microarrays (Riemer et al., 2004) and brain imaging
(Todd and Marois, 2004) where, again, effects may be sparse across a large sample of loci.

\section{Acknowledgement}

Partially supported by grants from EPSRC (EP/C51338X), (GR/S20574), (GR/S40443) and the Royal Society (J.F.).

\section{Appendix A}

Numbers inside $(\cdot)$ are electrode numbers (see Fig. 1). Digital numbers on the left hand side of an equality represent $x$-axis in Fig. 13, with concentration 1.

\section{A.1. Groups attained from real data}

$1=(05), 2=(13), 3=(15), 4=(16), 5=(25), 6=(26)$, $7=(35), 8=(36), 9=(41), 10=(43), 11=(44), 12=(45)$, $13=4614=(0515), 15=(1516), 16=(1525), 17=(16$ 26), $18=(2526), 19=(2535), 20=(2636), 21=\left(\begin{array}{l}35 \\ 3\end{array} 6\right)$, $22=(4344), 23=(3545), 24=(4445), 25=(3646), 26=$

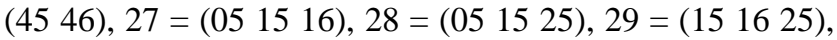

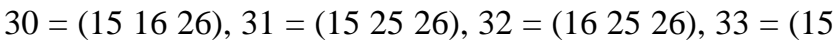
$2535), 34=(162636), 35=(252635), 36=(252636), 37$ $=(253536), 38=(263536), 39=(253545), 40=(3544$ $45), 41=(354546), 42=(434445), 43=(263646), 44=$ (35 3645$), 45=(353646), 46=(444546), 47=(051516$

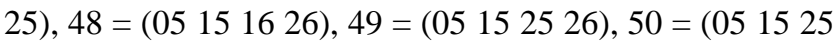

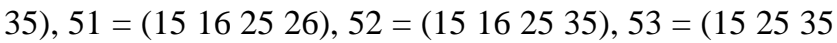

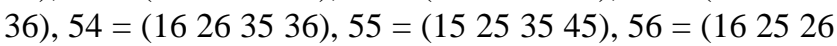

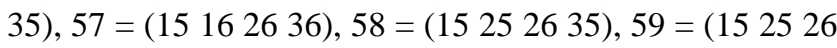
$36), 60=(16252636), 61=(25354445), 62=\left(\begin{array}{ll}35 & 4344\end{array}\right.$

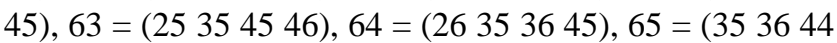

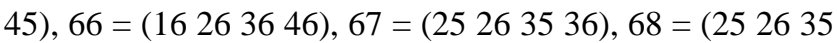

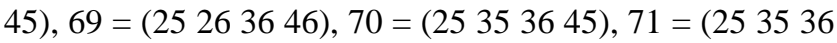
46), $72=(26353646), 73=\left(\begin{array}{l}35364546\end{array}\right), 74=\left(\begin{array}{l}35 \\ 3\end{array} 4445\right.$

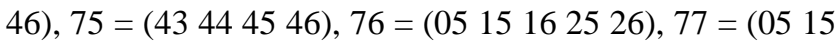

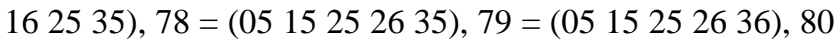

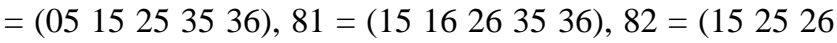
$3536), 83=(1625263545), 84=(1626353645), 85=$

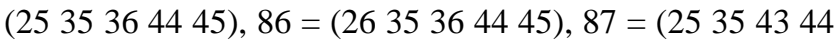
$45), 88=(2535444546), 89=(2636444546), 90=(35$

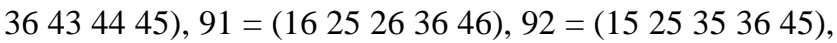

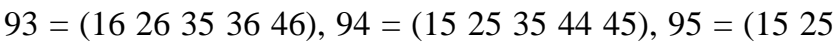
$353646), 96=(1525354546), 97=\left(\begin{array}{ll}16 & 25263536\end{array}\right), 98$

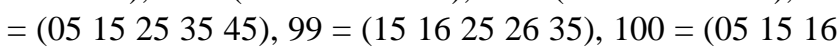

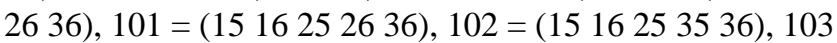
$=\left(\begin{array}{l}1516253545), 104=(1525263545), 105=(252635\end{array}\right.$ $3645), 106=(2526354445), 107=(1516263646), 108$ $=(1525263646), 109=(2526353646), 110=(252635$ $4546), 111=(2535364546), 112=(2635364546), 113$ $=(3536444546), 114=(3543444546), 115=(051516$ $252635), 116=(051516252636), 117=(0515162535$ 


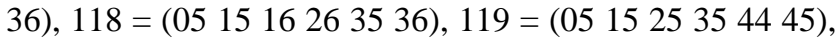

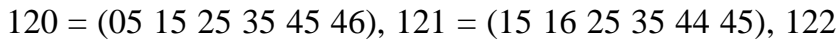
$=(151625354546), 123=(162635364445), 124=(15$ $2535434445), 125=(162636444546), 126=(252635$ $434445), 127=(051516263646), 128=\left(\begin{array}{ll}05152526 & 35\end{array}\right.$ $36), 129=\left(\begin{array}{ll}051525263545\end{array}\right), 130=\left(\begin{array}{ll}051525263646\end{array}\right)$,

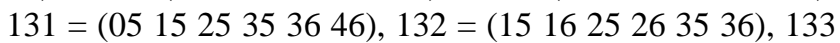
$=(051516253545), 134=(151625263545), 135=(15$ $1625353646), 136=(151626353645), 137=(152526$ $353645), 138=(152535364445), 139=(2526364445$ $46), 140=(253543444546), 141=(263536434445)$, $142=\left(\begin{array}{l}162635364546\end{array}\right), 143=\left(\begin{array}{l}253536434445\end{array}\right), 144$ $=(152535364546), 145=(162526353645), 146=(16$ $2526354445), 147=(252635364445), 148=(162526$ $353646), 149=(152526354445), 150=(1516252636$

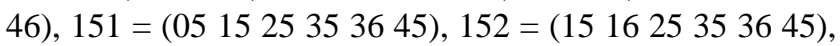

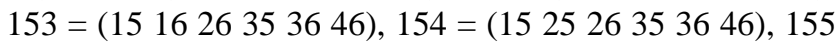
$=(152526354546), 156=(162526354546), 157=(25$ $2635364546), 158=(152535444546), 159=(252635$ $444546), 160=(253536444546), 161=(2635364445$ $46), 162=(263643444546), 163=\left(\begin{array}{l}353643444546\end{array}\right)$, $164=(05151625263536), 165=\left(\begin{array}{ll}05151625263545\end{array}\right)$, $166=\left(\begin{array}{l}05151625354445\end{array}\right), 167=\left(\begin{array}{l}05151625354546\end{array}\right)$, $168=\left(\begin{array}{l}05152526354445\end{array}\right), 169=\left(\begin{array}{l}05152526354546\end{array}\right)$, $170=(05152535364445), 171=\left(\begin{array}{l}15162535364445\end{array}\right)$,

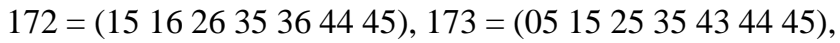
$174=\left(\begin{array}{ll}05152535444546\end{array}\right), 175=\left(\begin{array}{l}15162535434445\end{array}\right)$, $176=\left(\begin{array}{l}15162535444546\end{array}\right), 177=\left(\begin{array}{l}15162636444546\end{array}\right)$, $178=\left(\begin{array}{l}15252635434445\end{array}\right), 179=\left(\begin{array}{l}15252636444546\end{array}\right)$, $180=\left(\begin{array}{ll}16252636444546\end{array}\right), 181=\left(\begin{array}{l}15253536434445\end{array}\right)$, $182=\left(\begin{array}{l}16252635434445\end{array}\right), 183=\left(\begin{array}{l}16263536434445\end{array}\right)$, $184=(05151625263646), 185=\left(\begin{array}{l}05151625353645\end{array}\right)$, $186=(05151625353646), 187=(05151626353645)$, $188=(05151626353646), 189=\left(\begin{array}{l}05152526353645\end{array}\right)$, $190=(05152526353646), 191=(05152535364546)$, $192=(15162526353645), 193=\left(\begin{array}{l}15162526354445\end{array}\right)$, $194=\left(\begin{array}{l}15162535364546\end{array}\right), 195=\left(\begin{array}{l}16263536444546\end{array}\right)$, $196=\left(\begin{array}{l}15253543444546\end{array}\right), 197=\left(\begin{array}{l}16263643444546\end{array}\right)$, $198=(25263536434445), 199=\left(\begin{array}{l}15162635364546\end{array}\right)$, $200=\left(\begin{array}{l}15252635364445\end{array}\right), 201=\left(\begin{array}{l}15252635364546\end{array}\right)$,

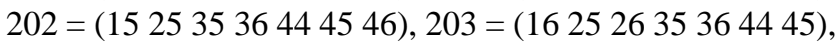

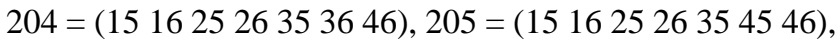
$206=(16252635364546), 207=\left(\begin{array}{l}15252635444546\end{array}\right)$, $208=\left(\begin{array}{l}16252635444546\end{array}\right), 209=\left(\begin{array}{l}25263536444546\end{array}\right)$, $210=(25263543444546), 211=\left(\begin{array}{l}25263643444546\end{array}\right)$, $212=\left(\begin{array}{l}25353643444546\end{array}\right), 213=\left(\begin{array}{l}26353643444546\end{array}\right)$,

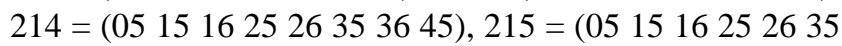
$4445), 216=(0515162526354546), 217=(05151625$ $35364445), 218=(0515162635364445), 219=(0515$ $252635364445), 220=(0515162535434445), 221=$ $(0515162535444546), 222=(0515162636444546)$, $223=(0515252635434445), 224=(051525263544$ $4546), 225=(0515252636444546), 226=(15162526$ $36444546), 227=(0515253536434445), 228=(0515$ $253536444546), 229=(1516253536444546), 230=$ (15 16263536444546$), 231=(0515253543444546)$,

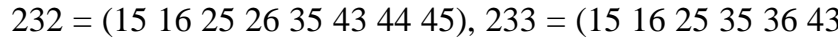
$4445), 234=(1516253543444546), 235=(15162635$ $36434445), 236=(1516263643444546), 237=(1525$ $263536434445), 238=(1525263643444546), 239=$ $(1625263643444546), 240=(1525353643444546)$,, $241=(1625263536434445), 242=(051516252635$ $3646), 243=(0515162535364546), 244=(05151626$ $35364546), 245=(0515252635364546), 246=(1516$ $252635364445), 247=(1516252635364546), 248=$ $(1516252635444546), 249=\left(\begin{array}{l}1525263536444546\end{array}\right)$,

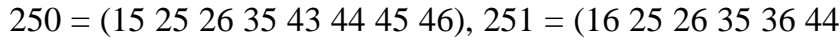
$4546), 252=(1625263543444546), 253=(16263536$ $43444546), 254=(2526353643444546), 255=(0515$ $16252635364445), 256=(051516252635434445)$, $257=(051516252635444546), 258=(0515162526$ $36444546), 259=(051516253536434445), 260=(05$ $1516253536444546), 261=(0515162635364445$ 46), $262=(051516263643444546), 263=(05152526$ $3536444546), 264=(051516253543444546), 265=$ $(051516263536434445), 266=(05152526353643$ $4445), 267=(051525263543444546), 268=(051525$ $263643444546), 269=(151625263643444546), 270$ $=(051525353643444546), 271=(15162526353643$ $4445), 272=(051516252635364546), 273=(151625$ $263536444546), 274=(151625263543444546), 275$ $=(151625353643444546), 276=(15162635364344$ $4546), 277=(152526353643444546), 278=(162526$ $353643444546), 279=(05151625263536434445)$, $280=(05151625263536444546), 281=(05151625$ $263543444546), 282=\left(\begin{array}{ll}05151625263643444546\end{array}\right)$, $283=(05151625353643444546), 284=(05151626$ $353643444546), 285=\left(\begin{array}{ll}05152526353643444546\end{array}\right)$, $286=\left(\begin{array}{ll}15 & 162526353643444546\end{array}\right), 287=(05151625$ $26353643444546),=\mathcal{Q}$.

\section{Appendix B}

\section{B.1. Groups attained from simulated data}

$1=(01), 2=(02), 3=(03), 4=(04), 5=(11), 6=(13), 7$ $=(21), 8=(22), 9=(23), 10=(24), 11=(31), 12=(33), 13$ $=(0102), 14=(0111), 15=(0203), 16=(0304), 17=(03$ $13), 18=(1121), 19=(2122), 20=(1323), 21=(2223)$, $22=(2324), 23=(2131), 24=(2333), 25=(010203), 26$ $=(011121), 27=(020304), 28=(020313), 29=(0304$ $13), 30=(112122), 31=(031323), 32=\left(\begin{array}{ll}13 & 22 \\ 23\end{array}\right), 33=$ (13 23 24), $34=(132333), 35=\left(\begin{array}{l}21 \\ 2223\end{array}\right), 36=\left(\begin{array}{l}22 \\ 2324\end{array}\right)$, $37=(112131), 38=(212231), 39=(222333), 40=(23$

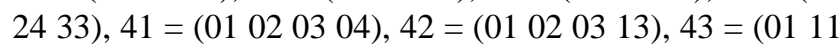
$2122), 44=\left(\begin{array}{lll}03 & 13 & 22\end{array} 23\right), 45=\left(\begin{array}{llll}11 & 21 & 22 & 23\end{array}\right), 46=\left(\begin{array}{ll}13 & 21\end{array}\right.$ 22 23 $), 47=\left(\begin{array}{llll}03 & 13 & 23 & 24\end{array}\right), 48=\left(\begin{array}{llll}21 & 22 & 23 & 24\end{array}\right), 49=\left(\begin{array}{ll}01 & 11\end{array}\right.$

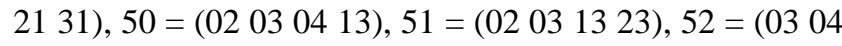
$1323), 53=\left(\begin{array}{llll}21 & 22 & 23 & 31\end{array}\right), 54=\left(\begin{array}{llll}03 & 13 & 23 & 33\end{array}\right), 55=\left(\begin{array}{ll}13 & 22\end{array}\right.$

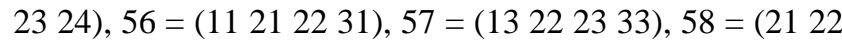

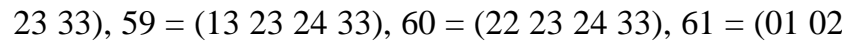




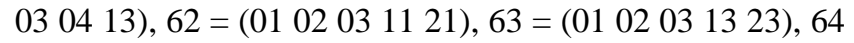

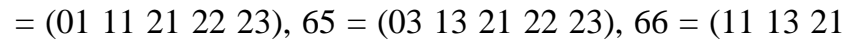

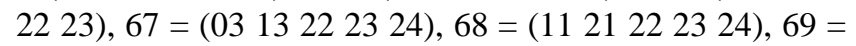
(13 21222324$), 70=(0111212231), 71=(02030413$

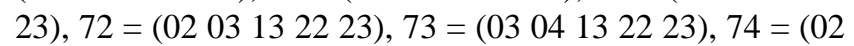

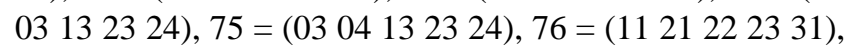

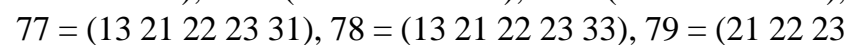
$2431), 80=(0203132333), 81=\left(\begin{array}{ll}0304 & 132333\end{array}\right), 82=$

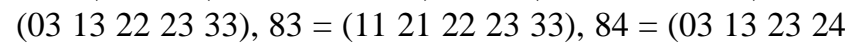

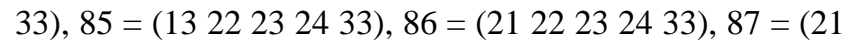
$22233133), 88=(01020341121), 89=(0102031113$

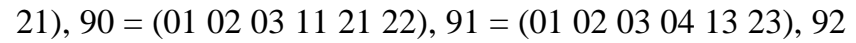
$=(010203132223), 93=(010203132324), 94=(0102$ $03132333), 95=(011113212223), 96=\left(\begin{array}{llll}01 & 11 & 21 & 22 \\ 03\end{array}\right.$

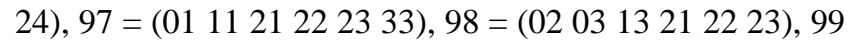

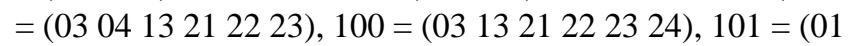

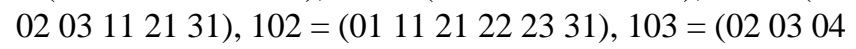

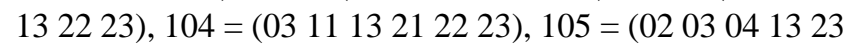

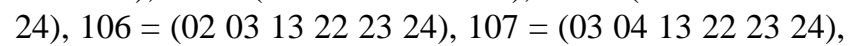

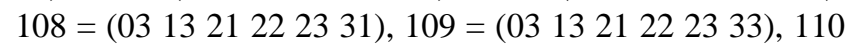

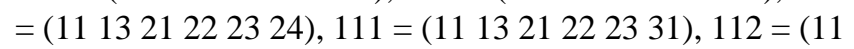

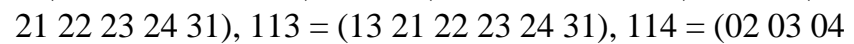
$132333), 115=(020313222333), 116=(0304132223$

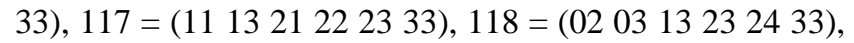

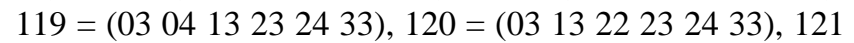

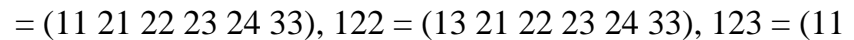

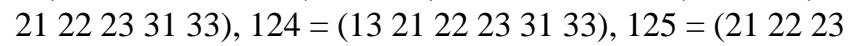
$243133), 126=(01020304111321), 127=(01020304$ $112122), 128=(01020304132223), 129=(01020304$ $132324), 130=(01020304132333), 131=(01020311$

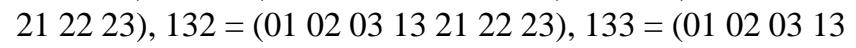

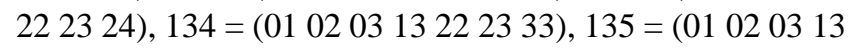
$232433), 136=\left(\begin{array}{llllll}01 & 11 & 1321222324\end{array}\right), 137=\left(\begin{array}{llll}01 & 11 & 1321\end{array}\right.$ $222331), 138=\left(\begin{array}{llllll}01 & 11 & 1322 & 22 & 23 & 33\end{array}\right), 139=\left(\begin{array}{llll}01 & 11 & 21 & 22\end{array}\right.$

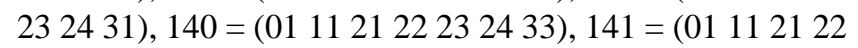
$233133), 142=(02030413212223), 143=(02031321$

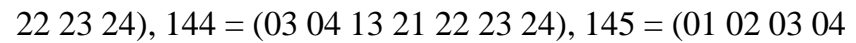
$112131), 146=(01020311132122), 147=(01020311$ $132123), 148=(01020311132131), 149=(01020311$

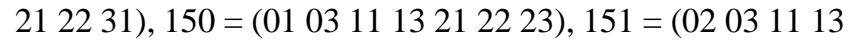

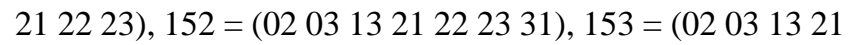
$222333), 154=(030411132122$ 23), $155=(02030413$ 2223 24), $156=(03041321222331), 157=(03041321$

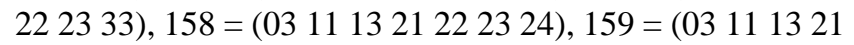

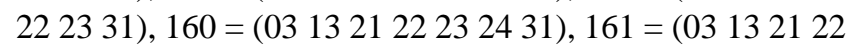

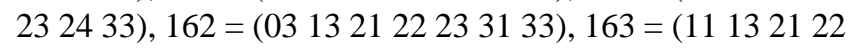
$232431), 164=(02030413222333), 165=\left(\begin{array}{llll}03 & 11 & 1321\end{array}\right.$ $222333), 166=\left(\begin{array}{lll}02 & 0304 & 13232433\end{array}\right), 167=\left(\begin{array}{lll}02 & 031322\end{array}\right.$ $232433), 168=\left(\begin{array}{lllll}03 & 04 & 1322 & 232433\end{array}\right), 169=\left(\begin{array}{llll}11 & 132122\end{array}\right.$

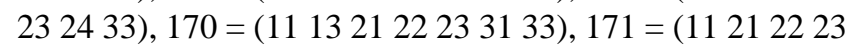

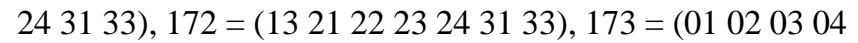
$11132122), 174=(0102030411132123), 175=(0102$ 0304112122 23), $176=\left(\begin{array}{ll}0102030413212223\end{array}\right), 177=$

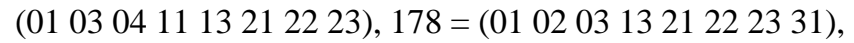
$179=(0102031113212333), 180=(010203041323$ $2433), 181=(0102031113212324), 182=(01020304$
$13222324), 183=(0102030413222333), 184=(0102$ 0311212223 24), $185=(0102031121222333), 186=$

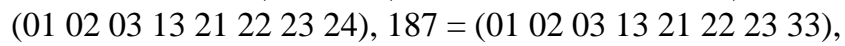

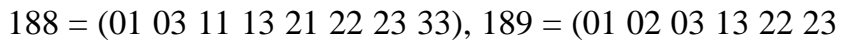
$2433), 190=(0103111321222324), 191=\left(\begin{array}{lll}01 & 11 & 1321\end{array}\right.$

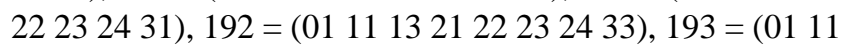
$132122233133), 194=(0111212223243133), 195=$ $(0203041321222324), 196=(0102030411132131)$,

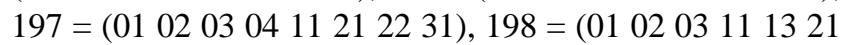
22 23), $199=(0102031113212231), 200=(01020311$ $13212331), 201=(0102031121222331), 202=(0203$ $041321222331), 203=(02030413212223$ 33), $204=$

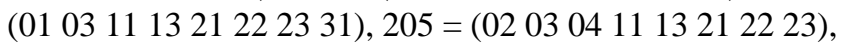

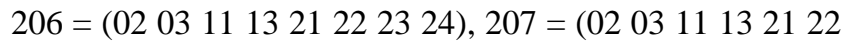
$2331), 208=(0203132122232431), 209=(02031321$ $22232433), 210=(0304132122232431), 211=(0304$ $132122232433), 212=(0203132122233133), 213=$

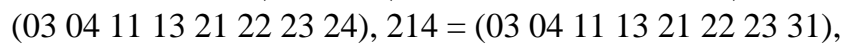

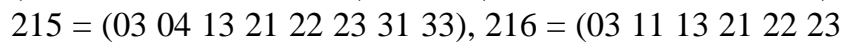
$2431), 217=(0203111321222333), 218=(03041113$ $21222333), 219=(0203041322232433), 220=(0311$ $132122232433), 221=(0311132122233133), 222=$

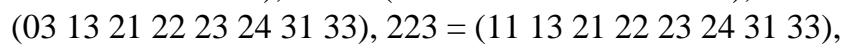
$224=(010203041113212223), 225=(0102030411$ $13212324), 226=(010304111321222324), 227=(01$

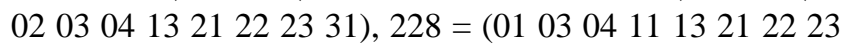
$31), 229=(010304111321222333), 230=(01020313$ $2122232431), 231=(010203041113212333), 232=$

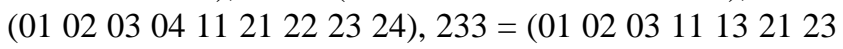
$2431), 234=(010203041121222333), 235=(010203$ $041321222324), 236=(010203041321222333), 237$

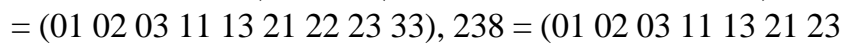
$2433), 239=(010203111321233133), 240=(010203$ $041322232433), 241=(010203111321222324), 242$

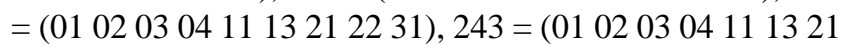
$2331), 244=(010203041121222331), 245=(010203$ $111321222331), 246=(010203112122232431), 247$

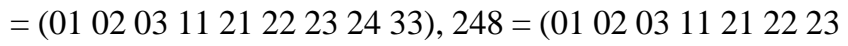
$3133), 249=(010203132122232433), 250=(010203$ $132122233133), 251=(010311132122232431), 252$

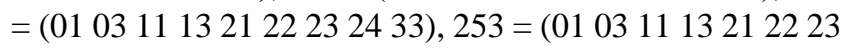

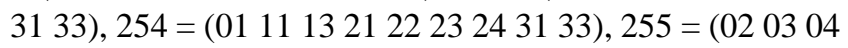
$111321222324), 256=(020304111321222331), 257$

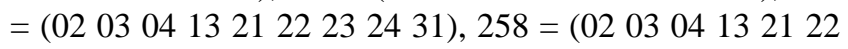
$232433), 259=(020304132122233133), 260=(0203$

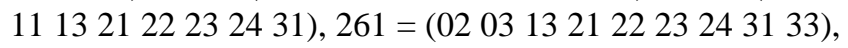

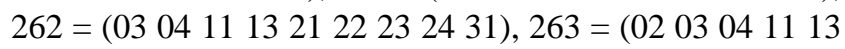
$21222333), 264=(020311132122232433), 265=(02$ $0311132122233133), 266=\left(\begin{array}{lll}0304 & 11 & 13 \\ 21 & 22 & 2324\end{array}\right.$ 33), $267=\left(\begin{array}{lllll}03 & 04 & 11 & 132122 & 233133\end{array}\right), 268=(03041321$

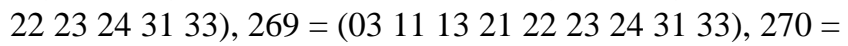
$(01020304111321222324), 271=(010203041113$ $21222331), 272=\left(\begin{array}{lll}01 & 02030411 & 1321232431\end{array}\right), 273=$

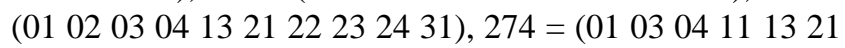
$22232431), 275=(01020304111321222333), 276=$ $(01020304111321232433), 277=\left(\begin{array}{llll}01 & 0304 & 11 & 1321\end{array}\right.$ 
$22232433), 278=\left(\begin{array}{lll}0102030411 & 1321233133\end{array}\right), 279=$ $\left(\begin{array}{llll}01020304112122 & 232431\end{array}\right), 280=\left(\begin{array}{llll}01020304 & 11 & 21\end{array}\right.$ $22232433), 281=(01020304112122233133), 282=$ $(01020304132122232433), 283=\left(\begin{array}{lllll}01020304 & 1321\end{array}\right.$ $22233133), 284=(01020311132122232431), 285=$

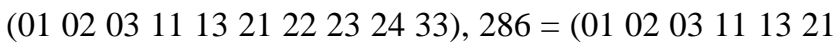

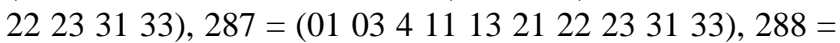

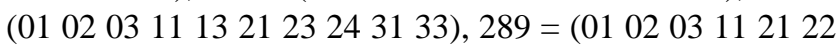
$23243133), 290=(01020313212223243133), 291=$

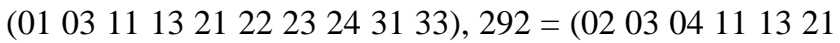
$22232431), 293=(02030411132122232433), 294=$

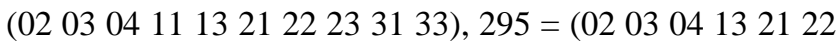
$23243133), 296=(02031113212223243133), 297=$

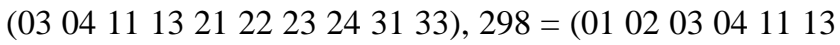

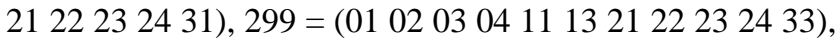

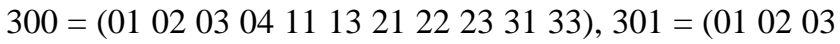

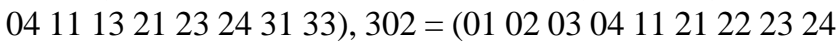
$3133), 303=(0102030413212223243133), 304=(01$ $02031113212223243133), 305=\left(\begin{array}{lll}010304 & 11 & 132122\end{array}\right.$ $23243133), 306=(0203041113212223243133), 307$ $=\left(\begin{array}{lllll}0102030411 & 132122 & 23243133\end{array}\right)$.

\section{Appendix C}

Here, we want to show theoretically that the fluctuations will become smaller as observed in MEANOVA results.

Let us look at $S_{2}(\mathcal{S})$,

$S_{2}(\mathcal{S})=-\left|I J(M-1)-\frac{|\mathcal{S}|+1-(J-1)}{2}\right| \ln \frac{\Lambda_{2}}{\chi_{(J-1)|\mathcal{S}|}^{2}(\alpha)}$

We could assume that $I J(M-1) / \chi_{(J-1)|\mathcal{S}|}^{2}(\alpha)$ is a constant when $|\mathcal{S}|$ is large. It is easily seen that

$$
\lim _{|\mathcal{S}| \rightarrow \infty} \frac{|\mathcal{S}|}{\chi_{(J-1)|\mathcal{S}|}^{2}(\alpha)}
$$

is a constant. For example, when $J=2, \alpha=0.05$, the constant is 1 . To assess the existence of limit, we need to consider the limit of $\Lambda_{2}$, which is more mathematically involved.

Let us assume that $S_{r}$ is a random, symmetric matrix. From a general results in random matrix (the Quarter circle law) (Cohen and Newman, 1984; Brody et al., 1981), we know that the asymptotic eigenvalue density function of $S_{r}$ converges in probability to

$P_{S_{r}}(x)=\frac{1}{2 \pi} \sqrt{\frac{4-x}{x}}, \quad 0<x<4$

We know that

$\left|S_{r}\right|=\lambda_{1} \ldots \lambda_{|\mathcal{S}|}$
Hence,

$\frac{\log \left|S_{r}\right|}{|\mathcal{S}|} \frac{\left[\log \lambda_{1}+\cdots+\log \lambda_{|\mathcal{S}|}\right]}{|\mathcal{S}|} \rightarrow E \log \lambda_{1}$

where $\lambda_{1}$ is distributed according to $P_{S_{r}}$. We then have

$\log \left|S_{r}\right| \sim|\mathcal{S}| E \log \lambda_{1}$

Similarly, for the matrix $S_{2}+S_{r}$, we conclude that

$\log \left|S_{r}+S_{2}\right| \sim|\mathcal{S}| \log E \bar{\lambda}_{1}$

where $\bar{\lambda}_{1}$ is the eigenvalue of the matrix $S_{r}+S_{2}$.

Combining all arguments above, we assert that the limit exists and so the fluctuation will become smaller and smaller.

\section{References}

Albright TD, Jessell TM, Kandel ER, Posner MI. Neural science: a century of progress and the mysteries that remain. Cell 2000;100:S1-55.

Bartletts MS. A note on the multiplying factors for various $\chi^{2}$ approximations. J R Stat Soc 1954;16:294-8.

Bracci E, Centonze D, Bernardi G, Calabresi P. Voltage-dependent membrane potential oscillations of rat striatal fast-spiking interneurons. J Physiol 2003;549:121-30.

Brody TA, Flores J, French JB, Mello PA, Pandey A, Wong SSM. Randommatrix physics: spectrum and strength fluctuations. Rev Mod Phys 1981;53:385-479.

Cohen JE, Newman CM. The stability of large random matrices and their products. Ann Probability 1984;12:283-310.

Davison A, Feng JF, Brown D. Dendrodendritic inhibition and odourinduced synchronization in a detailed olfactory bulb model. J Neurophysiol 2003;90:192-3.

Dayan P, Abbott LF. Theoretical neuroscience: computational and mathematical modeling of neural systems. MIT Press; 2001.

Debarbieux F, Audinat E, Charpak S. Action potential propagation in dendrites of rate mitral cells in vivo. J Neurosci 2003;23:5553-60.

Desmaisons D, Vincent JD, Lledo PM. Control of action potential timing by intrinsic subthreshold oscillations in olfactory bulb ouput neurons. J Neurosci 1999;19:10727-37.

Egger V, Svoboda K, Mainen ZF. Mechanisms of lateral inhibition in the olfactory bulb: efficiency and modulation of spike-evoked calcium influx into granule cells. J Neurosci 2003;23:7551-8.

Feller W. An introduction to probability theory and its application. New York: John Wiley and Sons; 1971.

Feng JF. Computational neuroscience: a comprehensive approach. Chapman and Hall/CRC Press; 2004.

Hunter JJ. Renewal theory in two dimensions: basic results. Adv Appl Probab 1974;6:376-91.

Hunter JJ. Renewal theory in two dimensions: asymptotic results. Adv Appl Probab 1974;6:546-62.

Johnson JL, Welsh JP. Independently movable multielectrode array to record multiple fast-spiking neurons in the cerebral cortex during cognition. Methods 2003;30:64-78.

Johnson RA, Wichern DW. Applied multivariate statistical analysis. 4th ed. Prentice Hall; 1988.

Kendrick KM, GuevaraGuzman R, Zorrilla J. Formation of olfactory memories mediated by nitric oxide. Nature 1997;388:670-4.

Margrie T, Schaefer A. Theta osciallation coupled spike latencies yield computational vigour in a mammalian sensory system. J Physiol 2003;546:363-74. 
Maynard EM, Hatsopoulos NG, Ojakangas CL, Acuna BD, Sanes JN, Normann RA, Donoghue JP. Neuronal interactions improve cortical population coding of movement direction. J Neurosci 1999;19:808393.

Neville KR, Haberly LB. Beta and gamma oscillations in the olfactory system of the urethane-anesthetized rat. J Neurophysiol 2003;90:3921-30.

Nicol A, Man MS, Feng JF, Mason R, Kendrick K. Differential spatial activation patterns evoled by odor stimui in the rate olfactory bulb. Annual Meeting of Neuroscience; 2003.

Patterson WR, Song YK, Bull CW, Ozden I, Deangellis AP, Lay C, McKay JL, Nurmikko AV, Donoghue JD, Connors BW. A microelectrode/microelectronic hybrid device for brain implantable neuroprosthesis applications. IEEE Trans Biomed Eng 2004;51:184553.
Pinato G, Midtgaard J. Regulation of granule cell excitability by a low-threshold calcium spike in turtle olfactory bulb. J Neurophysiol 2003;90:3341-51.

Riemer C, Neidhold S, Burwinkel M, Schwarz A, Schultz J, Kratzschmar J, Monning U, Baier M. Gene expression profiling of scrapieinfected brain tissue. Biochem Biophys Res Commun 2004;323:55664.

Todd JJ, Marois R. Capacity limit of visual short-term memory in human posterior parietal cortex. Nature 2004;428:751-4.

Uchida N, Mainen ZF. Speed and accuracy of olfactory discrimination in the rat. Nat: Neurosci 2003;1224-9.

Warwick K, Gasson M, Hutt B, Goodhew I, Kyberd P, Andrews B, Teddy P, Shad A. The application of implant technology for cybernetic systems. Arch Neurol 2004;60:1369-73. 